\title{
La violence faite aux adolescentes : un défi fondamental à la vraie égalité
}

Judith Bruce

Population Council

Follow this and additional works at: https://knowledgecommons.popcouncil.org/departments_sbsr-pgy

Part of the Family, Life Course, and Society Commons, Gender and Sexuality Commons, and the International Public Health Commons

How does access to this work benefit you? Let us know!

\section{Recommended Citation}

Bruce, Judith. 2012. "La violence faite aux adolescentes : un défı fondamental à la vraie égalité," GIRLS FIRST! Perspectives de programmation centrée sur les filles. New York: Population Council. 
UNE PUBLICATION GIRLS FIRST!

LA VIOLENCE

FAITE AUX

ADOLESCENTES :

UN DEFI

FONDAMENTAL

À LA VRAIE ÉGALITÉ

PAR JUDITH BRUCE 


\section{GIRLS FIRST!}

\section{PERSPECTIVES DE PROGRAMMATION CENTRÉE SUR LES FILLES}

La recherche et les programmes consacrés aux adolescentes se concentrent traditionnellement sur la sexualité, la santé génésique et le comportement, négligeant la problématique socioéconomique plus large qui sous-tend leurs droits humains, leur développement général, leur santé et leur bienêtre. De plus, les efforts d'amélioration de la vie des filles mettent souvent en vedette ceux et celles qui dominent ou influencent leur vie (les parents, la belle-famille, les garçons, les hommes, les agresseurs), sans prêter aux intéressées elles-mêmes l'attention qu'elles méritent en soi.

GIRLS FIRST! Perspectives de programmation centrée sur les filles est une série de cinq comptes rendus thématiques, rédigés par les experts et expertes du Population Council. Ces dossiers présentent un instantané de la base de connaissances telle qu'elle se présente, à un moment donné, dans ce domaine en évolution permanente. Ils abordent les cinq priorités stratégiques définies dans la Déclaration conjointe des Nations Unies, "Intensifier l'action menée pour promouvoir les droits des adolescentes " (mars 2010), symbole de l'engagement collectif de sept organismes de l'ONU au soutien des gouvernements et de leurs partenaires pour la promotion de politiques et programmes essentiels au profit des adolescentes les plus difficiles à atteindre. Ces comptes rendus

1. explorent l'évolution prochaine de l'éducation des filles;

2. définissent les grandes lignes d'approches innovantes en vue de l'amélioration de la santé des filles;

3. recadrent l'approche de la violence à l'encontre des filles;

4. décrivent les meilleurs moyens de cultiver les leaders féminines; et

5. expliquent de nouveaux modes inédits de collecte et d'exploitation des données relatives aux adolescentes.

Ils avancent des arguments novateurs en faveur de l'investissement dans les filles et mettent en valeur les pratiques prometteuses. Ils expriment un point de vue factuel progressiste quant aux allocations de ressources aptes à assurer le plus rapidement et le plus efficacement l'amélioration de la vie des filles.

Rédigés par des experts et expertes du Population Council, une organisation pionnière d'une recherche et programmation de pointe à l'intention des adolescentes vulnérables et marginalisées, ces rapports ont bénéficié, d'abord et surtout, de l'appui et du leadership de l'Équipe spéciale des Nations Unies pour les adolescentes. L'initiative a également reçu l'appui moral et financier d'ONU Femmes (anciennement UNIFEM), de la Nike Foundation, de la Fondation des Nations Unies et de la David and Lucile Packard Foundation. Ces comptes rendus viennent s'ajouter à la guidance programmatique et aux " boîtes à outils " aujourd'hui disponibles ; ils offrent une ressource de référence essentielle à toute initiative d'élaboration de politiques et programmes efficaces et durables au profit des filles. Notre espoir est qu'ils inspireront de nouvelles approches utiles aux efforts de réalisation des droits des adolescentes marginalisées du monde.

Avis

Ces comptes rendus sur la programmation pour adolescentes dans les domaines de l'éducation, de la santé, de la réduction de la violence, du leadership des filles et des données ont été préparés par le Population Council pour l'Équipe spéciale des Nations Unies pour les adolescentes, avec l'appui de la Fondation des Nations Unies, de la Nike Foundation, de la Packard Foundation et d'ONU Femmes.

Les opinions exprimées sont strictement celles des auteurs et ne reflètent pas nécessairement celles des partenaires donateurs.

(C) 2011 The Population Council, Inc. 


\title{
LA VIOLENCE FAITE AUX ADOLESCENTES : UN DÉFI FONDAMENTAL À LA VRAIE ÉGALITÉ
}

\author{
PAR JUDITH BRUCE
}

\section{CONNAISSANCES ET PRINCIPES FONDAMENTAUX _.........2}

La violence faite aux filles : une offense à la promesse d'égalité de la société

La violence extrême affecte au minimum une fille ou femme sur trois et une proportion élevée, peut-être même en hausse, de filles de moins de 15 ans.

L'acceptation de la violence mine l'estime personnelle des filles, les heures de leur journée et leur vie.....

Les cadres des droits humains sont souvent appliqués de manière axée sur les réalités des populations dominantes, en particulier masculines 5

En résumé : Les conséquences de la violence au niveau individuel et social sont si graves que la prévention doit être la toute première priorité.

\section{ÉTAT ACTUEL DES CHOSES}

Les programmes donnent la priorité au traitement et aux agresseurs plutôt qu'à la prévention et aux filles .8

Les programmes de jeunesse n'atteignent pas souvent les filles dans la mesure de leur besoin.

Un effort de recherche prometteur commence à définir les facteurs qui protègent les filles de la violence et les aide à voir et gérer clairement leur environnement .9

\section{POLITIQUES ET PRATIQUES PRÉCONISÉES}

Utiliser les données pour identifier les filles exceptionnellement vulnérables 10

Consacrer plus de ressources au renforcement de l'actifprotection des filles les plus vulnérables 10

Incorporer la connaissance des filles dans la conception des approches de prévention, d'atténuation, des procédures de déclaration et des programmes de traitement . .10

Créer des plates-formes et espaces régulièrement disponibles aux filles, en particulier celles les plus vulnérables à l'exploitation .11

Recruter intentionnellement les filles vulnérables .12

Ancrer les programmes autour des filles, en faisant d'elles les clientes principales : viser sélectivement les autres intervenants, en fonction de ce que pensent les filles. .12

Déterminer les médias éventuellement utiles en fonction de l'expérience des filles

Protéger les filles en quête de justice. .13

Mesurer les résultats au niveau des filles.

\section{RESSOURCES : PROGRAMMES « LABORATOIRES D'AP- PRENTISSAGE ", PUBLICATIONS ET OUTILS........................14}

Effort multiniveau de changement des normes de mariage précoce et de soutien des filles mariées : Berhane Hewan, Éthiopie

Girl spaces in school: Our girls, our future (Espaces Filles dans les écoles : nos filles, notre avenir) - effort de synergie pour mettre fin à la violence contre les filles en Zambie ..........14

Promotion d'espaces de travail sûrs pour les femmes employées dans les bars: Tanzanie .15

Biruh Tesfa (Avenir prometteur) : Offrir des espaces de rencontre, renforcer les compétences et l'actif-protection des filles employées comme domestiques, orphelines et migrantes en Éthiopie urbaine

Abriendo Oportunidades : Espaces sûrs au sein d'un programme destiné aux filles du Guatemala rural .....

Contrat de communauté contre la violence : Tostan, Afrique occidentale.

Programme Safe Cities : The Association for the Development and Enhancement of Women (association pour le développement et l'amélioration de la condition féminine), Égypte ........17

Paraprofessional Social Work Training Program (programme de formation paraprofessionnelle au travail social), International Rescue Committee (IRC), Région Somali d'Éthiopie ......17

Growing Up Safe and Healthy (SAFE) : Combler l'inégalité de genre par les messages de prévention de la violence de genre au Bangladesh..................................................................18

Apne Aap: Éliminer le trafic sexuel en Inde 18

Un SEUL programme : Approche pédagogique unifiée de la sexualité, du genre, du VIH et des droits humains

NOTES 


\section{CONNAISSANCES ET PRINCIPES FONDAMENTAUX}

La violence faite aux filles : une offense à la promesse d'égalité de la société

Les sociétés, riches comme pauvres, articulent chaque jour davantage des engagements qui garantissent aux filles un accès égal aux droits, aux services, à la participation sociale et à l'épanouissement économique. Pourtant, bien des menaces de violence - culturelle (mariage précoce, mutilation génitale des filles), intime (aux mains de membres de la famille et de partenaires), occasionnelle (aux mains d'étrangers) et planifiée (trafic) -, empêchent les filles de revendiquer leurs droits.

De nombreux pays ont ratifié la Convention sur l'élimination de toutes les formes de discrimination à l'égard des femmes (CEDF) et la Convention internationale des droits de l'enfant (CIDE), stipulant par ailleurs l'égalité entre les hommes et les femmes dans leurs constitutions respectives. Théoriquement, l'accès sûr aux ressources et aux aménagements est assuré à parts égales aux filles et aux garçons. Mais en réalité, une bien plus grande proportion de garçons et un sous-ensemble moindre de filles généralement privilégiées peuvent effectivement se prévaloir de leurs droits et opportunités. Le graphique ci-contre illustre de manière générale l'entrave que représente la violence à l'accès aux structures.

Le spectre de la violence conditionne souvent la vie des filles. Les actes de violence renforcent du reste le pouvoir exercé sur elles car l'opprobre de la violence s'attache plus à la fille qu'à l'agresseur. L'expérience de la violence est dévastatrice au niveau affectif et physique. Le pouvoir qu'elle a d'interrompre ou d'anéantir l'accès des filles aux droits, à la participation sociale et - décisivement - aux moyens de subsistance sûrs et corrects présente une raison tout aussi convaincante d'y mettre fin.

La violence est à ce point envahissante dans tant de sociétés qu'elle

\section{LA VIOLENCE ENTRAVE L'ACCÈS DES FILLES AUX SERVICES}

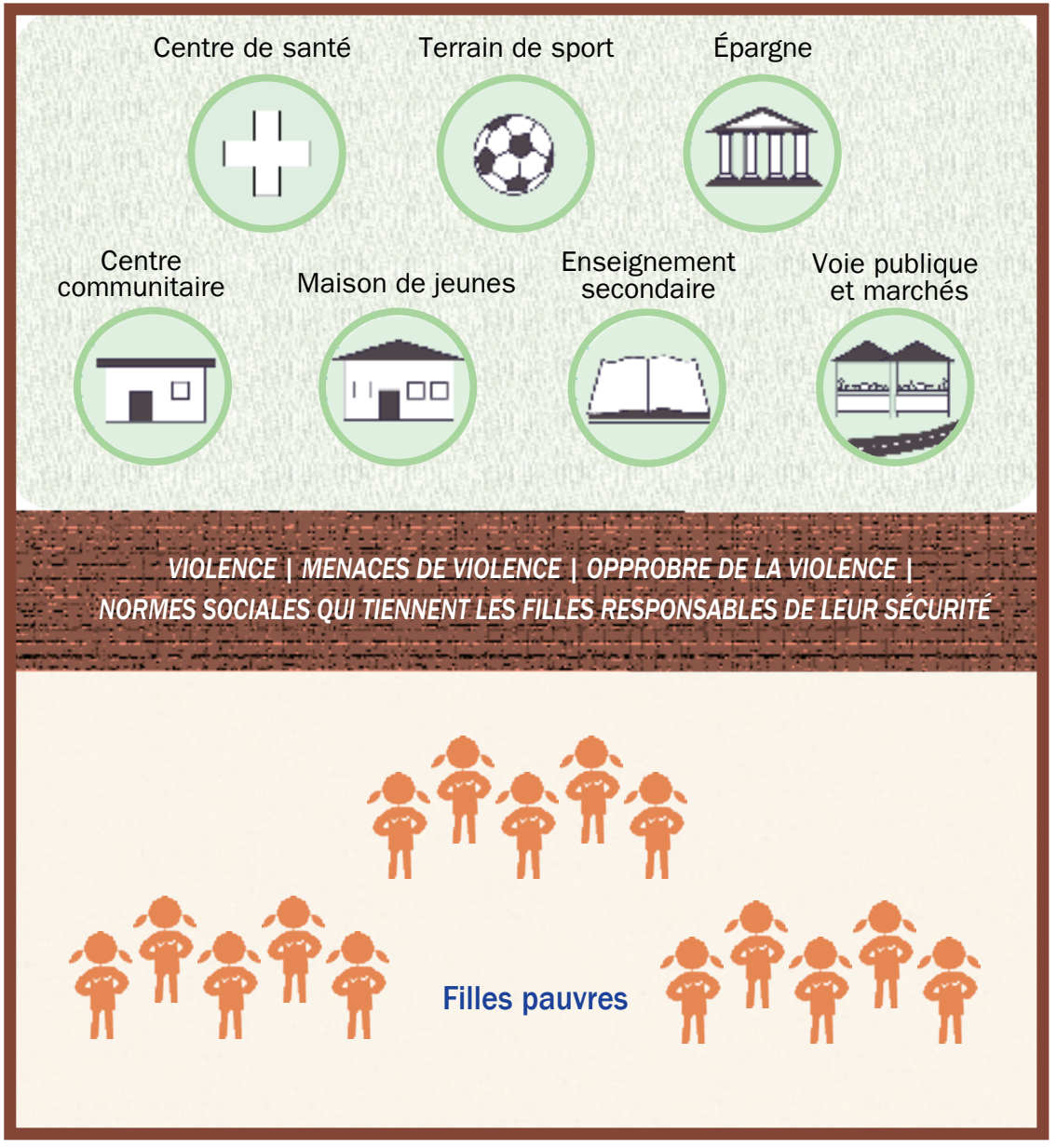

semble relever d'un " plan " actif ou même d'un effort d'opposition résolument contraire aux investissements consentis en faveur du bien-être des filles. Même en présence d'un certain progrès social, le " plan " de déni des droits des filles consiste notamment à les conditionner à éviter les opportunités qui s'offriraient sinon à elles pour gérer le risque. En intériorisant leur responsabilité de gérer ce risque, les filles précensurent leur potentiel.

La violence extrême affecte au minimum une fille ou femme sur trois et une proportion élevée, peut-être même en hausse, de filles de moins de 15 ans.

Un tiers des femmes du monde font état de violence physique (coups et blessures, viols et autres formes de maltraitance). Les auteurs en sont généralement des membres de leur famille ou leurs partenaires (Ellsberg et Heise $)^{1}$. Compte tenu du défi que représente la collecte de ce type de données sensibles, sous-déclaration comprise, on notera que les agressions sexuelles déclarées concernent en grande proportion, et souvent même en majorité, les filles de moins de 19 ans. Dans certains endroits, jusqu'à la moitié des agressions sexuelles déclarées sont commises sur des filles de moins de 15 ans. (Voir le Tableau 1.)

La proportion des filles affectées qui déclarent la violence sexuelle est extrêmement faible : dans certains contextes, environ la moitié seulement des filles qui la subissent en parlent à quelqu'un. (Les garçons hésitent aussi à signaler la violence sexuelle.) La démarche à suivre pour déclarer la 


\section{TABLEAU 1 PROPORTION ÉLEVÉE}

\section{D'AGRESSIONS SEXUELLES PARMI LES FILLES}

LIEU

Copperbelt, Zambie

Limpopo, Afrique du Sud

Malawi

Libéria

\section{PROFIL-ÂGE DES SURVIVANTES}

$49 \%$ de moins de 14 ans ; $85 \%$ de moins de 19 ans*.

$56 \%$ des victimes se présentant dans les centres de traumatologie sont des filles mineures et $31 \%$ sont des filles préadolescentes*.

Une étude nationale révèle que 50 \% des cas d'agression sexuelle d'enfants concernent des enfants âgés de 2 à 13 ans*.

La moitié des viols déclarés affectent des filles âgées de moins de 15 ans**.

*Keesbury, Jill et lan Askew. 2010. "Comprehensive responses to gender-based violence in lowresource settings: Lessons learned from implementation. " New York, NY: Population Council.

**Communications personnelles, Ministère de la Jeunesse et rencontre de Groupe de travail sur les adolescentes, Libéria, juin 2008.

violence peut retraumatiser les filles ${ }^{2}$. Dans certains contextes, l'accès aux services constitue un obstacle en soi car il n'existe au mieux dans de nombreuses communautés que de rares services organisés à l'intention des survivantes de la violence. Les autorités et les prestataires sont rarement formés à l'approche des cas de violence et encore moins à la manière de traiter les survivantes. Le doute et le blâme des prestataires peuvent causer de profonds dommages psychologiques chez les adolescentes et retarder ou empêcher le processus de guérison.

De récentes enquêtes menées par les gouvernements du Swaziland et de Tanzanie avec le soutien des US Centers for Disease Control and Prevention (CDC) et de I'UNICEF, documentent systématiquement la violence à l'encontre des enfants. Les niveaux de violence constatés sont extrêmement élevés. Dans les deux pays, environ un tiers des filles déclarent avoir subi des violences avant l'âge de 18 ans. Le rapport du Swaziland souligne que $40 \%$ des filles soumises à la violence le seront vraisemblablement encore (Reza) ${ }^{3}$. De plus, les auteurs de la violence se révèlent souvent être les proches des filles (principalement les hommes vivant sous le même toit ou dans le même quartier, avec un plus faible pourcentage d'enseignants ou d'autorités religieuses). Le lieu le plus fréquent de la violence est le foyer des filles. La violence aux mains d'étrangers représente moins de $15 \%$ de tous les actes perpétrés au Swaziland et $32 \%$ en Tanzanie 4 .

Le mariage précoce, l'une des formes les plus graves de la violence pourtant généralement exclue des statistiques de viol et de maltraitance - se pratique toujours dans des proportions choquantes dans certaines communautés. (Voir la Figure 1.) Si les tendances actuelles se poursuivent, plus de 100 millions de filles du monde en développement subiront cette rupture brutale de leur vie. Le mariage précoce les précipite dans l'esclavage sexuel, avec le haut risque d'issues négatives dont il s'accompagne, notamment les infections de l'appareil génital et la contamination par le VIH. Les filles victimes et rejetées de ce type d'union avant l'âge de 20 ans (les mariages précoces sont associés aux troubles matrimoniaux) courent un risque particulièrement élevé de contraction du $\mathrm{VIH}_{5}$.

Tandis que les jeunes femmes revendiquent plus d'autonomie dans leurs décisions sexuelles et matrimoniales, dans certaines situations émanant du contexte d'une " culture de rencontre moderne "(Jejeebhoy) ${ }^{6}$, elles peuvent se trouver amenées par la force ou par la ruse à avoir des rapports sexuels, sans disposer des compétences de négociation ou de la simple force physique qui leur permettraient de se protéger. Les Enquêtes démographiques et de santé (EDS) cherchent à savoir (dans beaucoup de pays mais pas dans tous) si la première rencontre sexuelle a été forcée ou imposée par la ruse. La prévalence des rapports sexuels non consensuels est souvent négligée lors de la conception des programmes d'éducation sexuelle conventionnelle.

Filles soumises à des premiers rapports sexuels forcés et grandes différences d'âge par rapport aux partenaires

L'importante différence d'âge avec son partenaire ou mari place souvent la fille en position désavantageuse pour éviter la violence et négocier un comportement sexuel sain. La différence d'âge entre les partenaires, mariés ou non, a des implications sur la capacité de la femme de négocier des rapports sexuels volontaires, sûrs et protégés. La recherche indique que les mariages entre des femmes jeunes et des hommes plus âgés sont moins équitables. (Voir le Tableau 2.)

\section{L'acceptation de la violence mine} l'estime personnelle des filles, les heures de leur journée et leur vie.

Le rôle de la violence dans la structure de la vie des filles au quotidien, de semaine en semaine et suivant la période de l'année est quantifiable. Les filles grandissent sous l'emprise de normes de mobilité réduite définies par la violence. La fille "décente " est soumise; le manque de soumission est réglé par la force, que la fille finit parfois par accepter comme justifiée. La soumission culturelle est profondément ancrée dans les traditions familiales, les relations conjugales asymétriques et les normes de la communauté. Elle est souvent appuyée, de manière directe et indirecte, par les politiques locales voire nationales ou les interprétations des grands principes religieux. 


\section{FIGURE 1 MARIAGE PRÉCOCE PARMI LES FILLES ÂGÉES DE 18 À 24 ANS AUX POINTS CHAUDS SOUS-NATIONAUX}

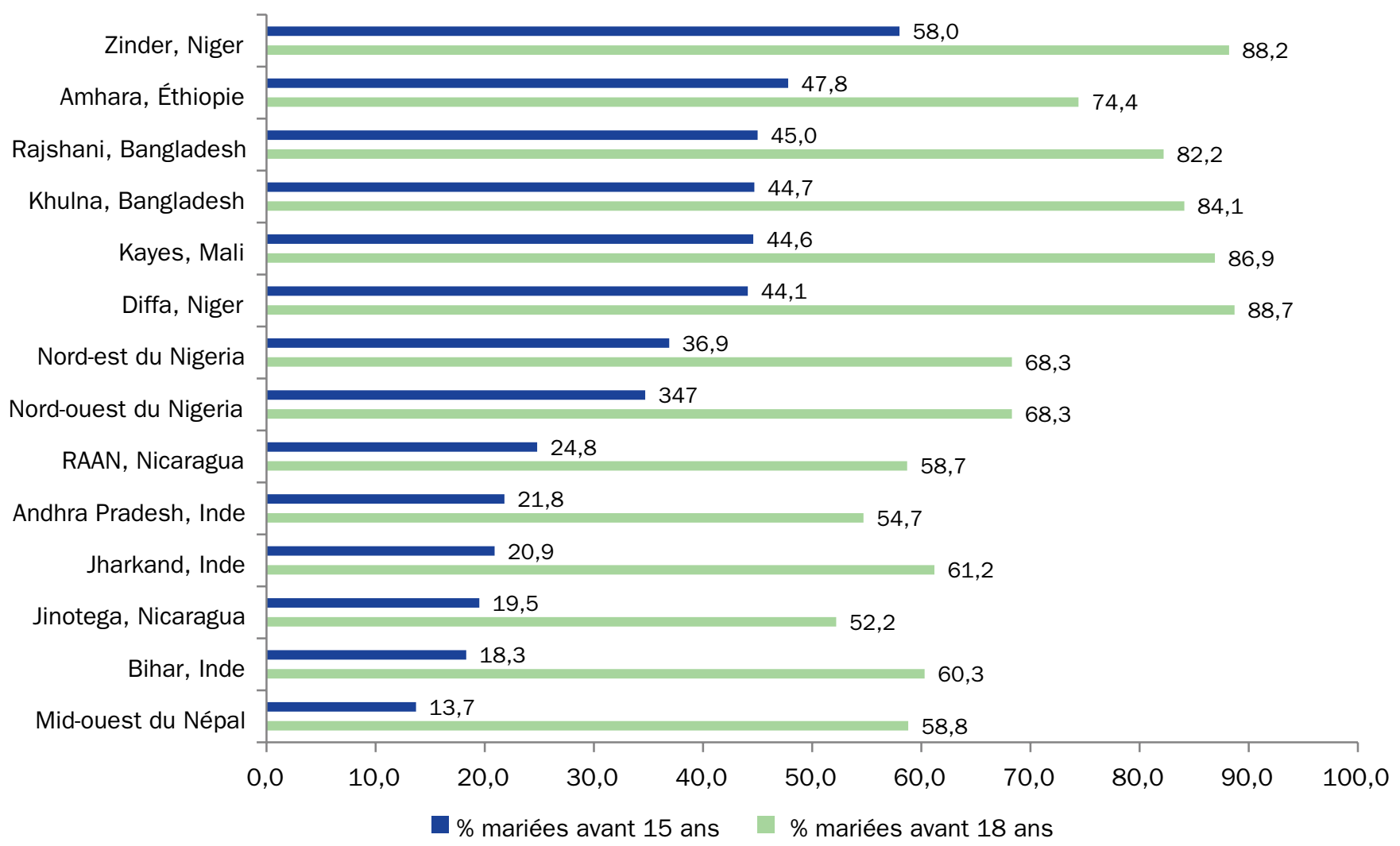

SOURCE : Données compilées par Adam Weiner, Population Council, août 2011. Source : Population Council. 2009. The Adolescent Experience In-Depth: Using Data to Identify and Reach the Most Vulnerable Young People. Rapports de pays. New York : Population Council. <http://www. popcouncil.org/ publications/serialsbriefs/AdolExplnDepth.asp>.

Les filles dont le sens de l'identité est faible dans les circonstances empiriquement violentes finissent par être conditionnées pour accepter et défendre l'usage de la force à leur encontre?. D'après les données des enquêtes EDS, de grandes proportions de filles justifient la violence qu'elles subissent pour une ou plusieurs raisons. Une proportion exorbitante la justifie même en toutes circonstances. (Voir le Tableau 3.)

\section{Justification et expérience de la violence}

Si les hommes et les garçons peuvent se trouver confrontés à un certain niveau de violence (en Tanzanie, $30 \%$ des filles, par rapport à environ $14 \%$ des garçons, subissent la violence sexuelle avant l'âge de 18 ans) ${ }^{8}$ et peuvent adapter tant soit peu leur structure de vie quotidienne pour améliorer leur sécurité, beaucoup de filles ne peuvent définir que de rares moments où elles se sentent parfaitement en sécurité et elles ne peuvent identifier pratiquement aucun espace où elles peuvent vivre en confiance. Des études systématiques (Hallman, Erulkar, Amin) ${ }^{9}$ produisent des ensembles de lieux fort différents où les jeunes hommes et femmes peuvent évoluer dans un contexte de sécurité et d'acceptation sociale. (Voir les Tableaux 4 et 5.)

Les mêmes 24 heures partagées par les jeunes hommes et femmes paraissent bien différentes : dans certains contextes, les filles ne pouvent pas même identifier une heure où elles se sentent en sécurité ${ }^{10}$. La sécurité des filles, en termes absolus et par rapport aux hommes, au sein du ménage, dans la communauté et à l'école, est quantifiable, de même que la peur de harcèle- ment (Hallman, Erulkar, Amin) ${ }^{11}$. Les évaluations de sécurité (Bruce, Austrian) ${ }^{12}$ proposées dans le contexte d'ateliers ou d'enquêtes formelles peuvent révéler les perceptions de sécurité des filles de différentes catégories sociales (elles permettent aussi la comparaison systématique par rapport aux garçons).

Une étude des stratégies adoptées par les filles de Zambie pour se protéger du VIH est révélatrice ${ }^{13}$. Sans doute les observations les plus frappantes ne sont-elles pas les hauts niveaux de violence (plus du tiers des filles connaissent une fille violentée par un enseignant et près de la moitié en connaissent une violentée par un membre masculin intime du foyer) mais le fait que $86 \%$ soulignent que leur réponse à cette violence a été incorporée dans leurs plans journaliers et que cette réponse consiste à " rester chez 


\section{TABLEAU 2 DIFFÉRENCE D'ÂGE PAR RAPPORT AU PARTENAIRE ET VULNÉRABILITÉ DES FILLES}

\begin{tabular}{|c|c|c|c|c|c|c|c|}
\hline \multirow[b]{2}{*}{ PAYS } & \multirow[b]{2}{*}{ ANNÉE EDS } & \multicolumn{2}{|c|}{$\begin{array}{c}\text { \% DES FILLES DE } 15 \text { À } 24 \\
\text { ANS DONT LE PREMIER } \\
\text { RAPPORT SEXUEL } \\
\text { A ÉTÉ FORCÉ }\end{array}$} & \multicolumn{2}{|c|}{$\begin{array}{c}\text { \% DES FILLES DE } 15 \text { À } 24 \text { ANS } \\
\text { DONT LA DIFFÉRENCE D'ÂGE } \\
\text { PAR RAPPORT AU PREMIER } \\
\text { PARTENAIRE SEXUEL ÉTAIT } \\
\text { D'AU MOINS } 10 \text { ANS }\end{array}$} & \multicolumn{2}{|c|}{$\begin{array}{c}\text { \% DES FILLES DE } 15 \text { À } 24 \\
\text { ANS DONT LA DIFFÉRENCE } \\
\text { D'ÂGE PAR RAPPORT AU MARI/ } \\
\text { PARTENAIRE ACTUEL EST D'AU } \\
\text { MOINS } 10 \text { ANS }\end{array}$} \\
\hline & & NATIONAL & POINT CHAUD & NATIONAL & POINT CHAUD & NATIONAL & POINT CHAUD \\
\hline Bénin & 2006 & 27,2 & $\begin{array}{c}\text { 47,6 } \\
\text { (Ouémé) }\end{array}$ & 18,9 & $\begin{array}{c}32,0 \\
\text { (Atacora) }\end{array}$ & 30,2 & $\begin{array}{c}43,5 \\
\text { (Donga) }\end{array}$ \\
\hline $\mathrm{RDC}$ & 2007 & 63,8 & $\begin{array}{c}84,4 \\
\text { (Maniema) }\end{array}$ & 14,9 & $\begin{array}{c}25,3 \\
\text { (Kasai Oriental) }\end{array}$ & 26,2 & $\begin{array}{c}35,6 \\
\text { (Kasai Oriental) }\end{array}$ \\
\hline Inde & 2005-06 & $\mathrm{s} / \mathrm{o}$ & $\mathrm{s} / \mathrm{o}$ & 14,8 & $\begin{array}{c}42,4 \\
\text { (Assam) }\end{array}$ & 13,6 & $\begin{array}{c}\text { 34,6 } \\
\text { (Assam) }\end{array}$ \\
\hline Libéria & 2007 & 9,5 & $\begin{array}{c}13,3 \\
\text { (Sud-Est A) }\end{array}$ & 38,4 & $\begin{array}{c}53,8 \\
\text { (Sud-Est A) }\end{array}$ & 30,3 & $\begin{array}{c}35,6 \\
\text { (Sud-Est A) }\end{array}$ \\
\hline Mali & 2006 & 24,9 & $\begin{array}{c}33,4 \\
\text { (Sikasso) }\end{array}$ & 45,5 & $\begin{array}{c}\text { 65,0 } \\
\text { (Tombouctou) }\end{array}$ & 53,4 & $\begin{array}{c}\text { 73,0 } \\
\text { (Tombouctou) }\end{array}$ \\
\hline Népal & 2006 & s/o & s/o & 3,0 & $\begin{array}{c}5,4 \\
\text { (Occidental) }\end{array}$ & 8,1 & $\begin{array}{c}11,7 \\
\text { (Occidental) }\end{array}$ \\
\hline Rwanda & 2005 & s/o & $\mathrm{s} / \mathrm{o}$ & 27,0 & $\mathrm{~s} / \mathrm{o}$ & 17,3 & $\begin{array}{c}30,9 \\
\text { (Ville de Kigali) }\end{array}$ \\
\hline Ouganda & 2006 & 25,2 & $\begin{array}{c}35,2 \\
\text { (Sud-Ouest) }\end{array}$ & 17,5 & $\begin{array}{c}28,6 \\
\text { (Occidental) }\end{array}$ & 20,1 & $\begin{array}{c}27,9 \\
\text { (Centre-Est) }\end{array}$ \\
\hline Zambie & 2007 & $\mathrm{~s} / \mathrm{o}$ & $\mathrm{s} / \mathrm{o}$ & 7,8 & $\begin{array}{l}14,2 \\
\text { (Nord) }\end{array}$ & 15,5 & $\begin{array}{c}17,3 \\
\text { (Copperbelt) }\end{array}$ \\
\hline
\end{tabular}

SOURCE : Données compilées par Adam Weiner, Population Council, août 2011. Source : Population Council. 2009. The Adolescent Experience In-Depth: Using Data to Identify and Reach the Most Vulnerable Young People. Rapports de pays. New York : Population Council. <http://www.popcouncil.org/ publications/serialsbriefs/AdolExplnDepth.asp>.

soi ". Le retrait des filles des opportunités, leur tendance à se protéger en limitant leurs options et l'élimination de parties entières de leur environnement (le marché, l'industrie, l'emploi, les droits sociaux dans les cliniques de santé) tiennent tous à leurs craintes en ce qui concerne la sécurité.

Les cadres des droits humains sont souvent appliqués de manière axée sur les réalités des populations dominantes, en particulier masculines.

Les tentatives officielles de protection des filles et des femmes face à la violence - notamment les cadres des droits humains et les politiques et procédures de réduction de la violence - omettent souvent les infrastructures protectrices et de soutien dont dispose le sexe masculin mais pas le féminin. Les filles et les femmes, dans une mesure largement inférieure à celle des hommes, ne peuvent se rencontrer librement et régulièrement les unes les autres, car leurs mouvements sont contrôlés par autrui. Les endroits où les filles et les femmes risquent le plus de subir la violence sont les espaces intimes et ceux qui ne présentent qu'une protection nominale. Les auteurs les plus vraisemblables de la violence et les adultes complices sont porteurs de responsabilités (par exemple, les membres masculins du ménage ou du quartier, ceux qui seraient sinon des partenaires romantiques, les parents, enseignants, employeurs, autorités légales et parfois même des représentants religieux).

En résumé : Les conséquences de la violence au niveau individuel et social sont si graves que la prévention doit être la toute première priorité.

L'expérience individuelle de la violence a des conséquences physiques, psy- 


\section{TABLEAU 3 JUSTIFICATION ET EXPÉRIENCE DE LA VIOLENCE}

\begin{tabular}{|c|c|c|c|c|c|c|}
\hline \multirow[b]{2}{*}{$\begin{array}{l}\text { REGION/ } \\
\text { PAYS }\end{array}$} & \multirow[b]{2}{*}{$\begin{array}{c}\text { ANNÉE } \\
\text { EDS }\end{array}$} & \multicolumn{2}{|c|}{$\begin{array}{l}\text { \% DES FILLES DE } 15 \text { À } 24 \text { ANS } \\
\text { QUI PENSENT QUE LA VIOLENCE } \\
\text { CONJUGALE EST ACCEPTABLE DANS } \\
\text { CERTAINES CIRCONSTANCES }\end{array}$} & \multicolumn{3}{|c|}{$\begin{array}{l}\text { \% DES FILLES DE } 15 \text { À } 24 \text { ANS } \\
\text { SOUMISES À LA VIOLENCE PHYSIQUE } \\
\text { DEPUIS L'ÂGE DE } 15 \text { ANS }\end{array}$} \\
\hline & & NATIONAL & $\begin{array}{l}\text { POINT } \\
\text { CHAUD }\end{array}$ & NATIONAL & $\begin{array}{l}\text { POINT } \\
\text { CHAUD }\end{array}$ & $\begin{array}{l}\text { POINT CHAUD } \\
\text { (MARIÉES OU } \\
\text { L'AYANT DÉJA ÉTÉ) }\end{array}$ \\
\hline \multicolumn{7}{|c|}{ AFRIQUE SUBSAHARIENNE } \\
\hline Bénin & 2006 & 44,6 & $\begin{array}{c}74,2 \\
\text { (Atacora) }\end{array}$ & $\mathrm{s} / \mathrm{o}$ & $\mathrm{s} / \mathrm{o}$ & s/o \\
\hline RDC & 2007 & 82,6 & $\begin{array}{c}89,3 \\
\text { (Équateur) }\end{array}$ & 59,7 & $\begin{array}{c}75,4 \\
\text { (Équateur) }\end{array}$ & $\begin{array}{c}88,4 \\
\text { (Kinshasa) }\end{array}$ \\
\hline Libéria & 2007 & 60,6 & $\begin{array}{c}74,4 \\
\text { (Centre-Nord) }\end{array}$ & 32,5 & $\begin{array}{c}40,5 \\
\text { (Monrovia) }\end{array}$ & $\begin{array}{c}48,1 \\
\text { (Monrovia) }\end{array}$ \\
\hline Mali & 2006 & 77,3 & $\begin{array}{c}88,9 \\
\text { (Kayes) }\end{array}$ & $\mathrm{s} / \mathrm{o}$ & $\mathrm{s} / \mathrm{o}$ & $\mathrm{s} / \mathrm{o}$ \\
\hline Rwanda & 2005 & 50,6 & $\begin{array}{c}71,9 \\
\text { (Cyangugu) }\end{array}$ & 22,9 & $\begin{array}{c}35,7 \\
\text { (Umutara) }\end{array}$ & $\begin{array}{c}48,7 \\
\text { (Ville de Kigali) }\end{array}$ \\
\hline Ouganda & 2006 & 72,4 & $\begin{array}{c}87,7 \\
\text { (Ouest de Nile) }\end{array}$ & 55,4 & $\begin{array}{l}71,3 \\
\text { (Est) }\end{array}$ & $\begin{array}{l}73,5 \\
\text { (Est) }\end{array}$ \\
\hline Zambie & 2007 & 63,1 & $\begin{array}{c}83,5 \\
\text { (Luapula) }\end{array}$ & 40,6 & $\mathrm{~s} / \mathrm{o}$ & $\begin{array}{c}59,5 \\
\text { (Copper Belt) }\end{array}$ \\
\hline \multicolumn{7}{|l|}{ ASIE } \\
\hline Bangladesh* & 2007 & 31,4 & $\begin{array}{c}47,5 \\
\text { (Barisal) }\end{array}$ & 26,7 & N/A & $\begin{array}{c}36,7 \\
\text { (Rajshahi) }\end{array}$ \\
\hline Cambodge & 2005 & 55,6 & $\begin{array}{c}84,9 \\
\text { (Svay Rieng) }\end{array}$ & 10,1 & $\begin{array}{c}24,3 \\
\text { (Pursat) }\end{array}$ & N/A \\
\hline Inde & $2005-06$ & 47,3 & $\begin{array}{c}83,2 \\
\text { (Manipur) }\end{array}$ & 14,7 & $\begin{array}{c}28,8 \\
\text { (Tripura) }\end{array}$ & $\begin{array}{c}41,1 \\
\text { (Bengal Occidental) }\end{array}$ \\
\hline Indonésie* & 2002-03 & 29,6 & $\begin{array}{c}55,5 \\
\text { (W. Nusa Tenggara) }\end{array}$ & $\mathrm{s} / \mathrm{o}$ & $\mathrm{s} / \mathrm{o}$ & $\mathrm{s} / \mathrm{o}$ \\
\hline Népal & 2006 & 23,6 & $\begin{array}{c}\text { 30,6 } \\
\text { (Mid-ouest) }\end{array}$ & $\mathrm{s} / \mathrm{o}$ & $\mathrm{s} / \mathrm{o}$ & $\mathrm{s} / \mathrm{o}$ \\
\hline Philippines & 2003 & 24,8 & $\begin{array}{c}66,1 \\
\text { (ARMM) }\end{array}$ & $\mathrm{s} / \mathrm{o}$ & $\mathrm{s} / \mathrm{o}$ & $\mathrm{s} / \mathrm{o}$ \\
\hline
\end{tabular}

*Enquête des femmes mariées ou l'ayant jamais été.

SOURCE : Données compilées par Adam Weiner, Population Council, août 2011. Source : Population Council. 2009. The Adolescent Experience In-Depth: Using Data to Identify and Reach the Most Vulnerable Young People. Rapports de pays. New York : Population Council. <http://www.popcouncil.org/ publications/serialsbriefs/AdolExplnDepth.asp>.

chologiques, sexuelles et économiques dévastatrices durables - à l'échelle d'une vie et d'une génération à l'autre - bien documentées.

L'étude de la violence au niveau individuel peut être conceptualisée utilement comme une attaque à un " actif " fondamental - de capital humain et de dignité - dont on ne peut peut-être jamais "se relever " vraiment. Si beaucoup de femmes reconquièrent courageusement une vie appréciée, une partie considérable de la population ne retrouve pas pleinement sa confiance et son intégrité physique. Longtemps après le traitement ou la réparation de leur corps, les survivantes de la violence peuvent éprouver des 
TABLEAU 4 ACCÈS RELATIF DES FILLES AUX FILETS DE SÉCURITÉ SOCIAUX ET NIVEAU D’AISE DANS LES COMMUNAUTÉS (QEBELÉS) AUX ALENTOURS DE LA GARE D'AUTOBUS D'ADDIS ABEBA

INDICATEURS DE VULNÉRABILITÉ ET SENS DE BIEN-ÊTRE, PAR SEXE ET SITUATION D'EMPLOI

\begin{tabular}{|c|c|c|c|c|c|}
\hline & \multicolumn{2}{|c|}{ GARÇONS } & \multicolumn{3}{|c|}{ FILLES } \\
\hline $\begin{array}{l}\text { CATÉGORIE } \\
\text { DE VULNÉRABILITÉ }\end{array}$ & $\begin{array}{l}\text { SANS EMPLOI } \\
\qquad(\mathrm{N}=\mathbf{2 8 5})\end{array}$ & $\begin{array}{l}\text { EMPLOYÉS } \\
(\mathrm{N}=111)\end{array}$ & $\begin{array}{l}\text { SANS EMPLOI } \\
\qquad(N=545)\end{array}$ & $\begin{array}{c}\text { EMPLOYÉES - NON } \\
\text { DOMESTIQUES } \\
(\mathrm{N}=30)\end{array}$ & $\begin{array}{c}\text { EMPLOYÉES } \\
\text { DOMESTIQUES } \\
(\mathbf{N}=99)\end{array}$ \\
\hline Aucune scolarité & 0,7 & 4,5 & 4,3 & 13,3 & $37,8 * * *$ \\
\hline Ne vivent pas avec leurs parents & 26,0 & 41,4 & 45,9 & 66,7 & $97,0 * * *$ \\
\hline Migration à Addis & 18,9 & 35,1 & 35,1 & 46,7 & $97,0 * * *$ \\
\hline Nombreux amis & 62,4 & 51,4 & 26,9 & 23,2 & $7,1 * * *$ \\
\hline $\begin{array}{l}\text { Peur d'être victime de la violence } \\
\text { dans le quartier }\end{array}$ & 13,9 & 20,7 & 30,3 & 26,7 & $39,4 * * *$ \\
\hline
\end{tabular}

Différences entre les groupes significatives à *<0,05; **p<0,01; ***p<0,001.

SOURCE : Erulkar A. et Mekbib T. 2007. "Invisible and vulnerable: Adolescent domestic workers in Addis Ababa, Ethiopia ", Vulnerable Child and Youth Studies 2(3): 246-256.

\section{TABLEAU 5 SOUTIEN COMMUNAUTAIRE, PERCEPTIONS DE SÉCURITÉ ET EXPÉRIENCE DE LA CRIMINALITÉ, PAR SEXE}

\section{ÉNONCÉ}

\section{GARÇONS}

FILLES

$(\mathrm{N}=400)$

$(\mathrm{N}=676)$

Expérience du soutien communautaire

dans le quartier

Tu as beaucoup d'amis dans le quartier

$59,0 * * *$

23,8

Si tu n'avais pas d'endroit où passer la nuit, quelqu'un de ton quartier t'accepterait chez lui ou chez elle

$33,3 * * *$

Si tu avais besoin d'argent rapidement, quelqu'un dans ton quartier pourrait t'en prêter

$22,0 *$

Tu as un endroit dans le quartier (en dehors de chez toi ou de l'école) ou tu peux rencontrer tes amis ou amies

$47,5 * * *$

Différences entre les groupes significatives à $*<0,05 ; * * p<0,01 ; * * * p<0,001$.

SOURCE : Erulkar, Annabel. "Adolescent Life in Low Income and Slum Areas of Addis Ababa, Ethiopia. " 2004. Population Council. difficultés à retrouver un sentiment de contrôle de leur vie.

Les survivantes de la violence souffrent de séquelles durables sur leur santé, notamment des syndromes de douleurs chroniques, des problèmes d'abus d'alcool et de drogue, des grossesses non désirées, des complications obstétricales, des infections sexuellement transmissibles (y compris le VIH), des taux de morbidité et de mortalité supérieurs, des problèmes de santé mentale, des problèmes gynécologiques et une moindre aptitude à fonctionner (Campbell) ${ }^{14}$. Les effets socioéconomiques se reflètent dans un absentéisme accru, une moindre participation à la vie active et une productivité réduite. La capacité des filles de gagner un salaire proportionné à leurs compétences et d'assumer le contrôle physique de leur revenu en est affaiblie. Une étude réalisée récemment au Swaziland, impressionnante par la profondeur et l'envergure de la recherche, conclut que les effets à court et à long terme de la violence sont à ce point dévastateurs que la priorité doit être donnée, indubitablement, à la prévention (Reza) $)^{15}$. 


\section{ÉTAT ACTUEL DES CHOSES}

Les programmes donnent la priorité au traitement et aux agresseurs plutôt qu'à la prévention et aux filles.

Cette approche correspond à celle ancrée dans les droits humains, où la priorité va à l'engagement des individus le plus directement affectés. Beaucoup de programmes de prévention de la violence de genre, y compris ceux qui soulignent le jeune âge auquel interviennent de nombreuses violations, planifient davantage l'engagement avec les symboles d'autorité et les responsables de la violence qu'avec les filles elles-mêmes. Ils œuvrent " pour le compte des filles " mais pas avec les filles. Ces initiatives en soi semblent en valoir la peine mais, considérées dans leur contexte, elles peuvent être dotées de ressources excessives par rapport aux besoins pratiques et stratégiques. Voici peu, un grand pays d'Afrique subsaharienne a adopté un programme ambitieux de prévention de la violence de genre, notant qu'une très grande proportion de cette violence concernait les adolescentes et les jeunes femmes. Le plan proposé allouait cependant moins de $5 \%$ des ressources totales à l'engagement direct des populations jeunes, mélangeant garçons et filles dans les groupes bénéficiaires, et il n'avait pas défini de jalons de résultat distincts pour chaque sexe. Le Tableau 6 donne une idée de l'allocation actuelle des ressources et des groupes principalement engagés.

Les programmes de jeunesse n'atteignent pas souvent les filles dans la mesure de leur besoin.

Beaucoup de programmes de santé infantile, de participation civique, de développement social et, tout particulièrement, de jeunesse, ont été organisés au nom de la résolution de la vulnérabilité des jeunes en fin d'enfance et début d'adolescence, pour leur donner un "filet de sécurité " et une filière d'accès à la participation civique. Étonnamment, les segments de la population infantile et adolescente qui courent le plus grand risque et dotés du moindre capital social sont les moins susceptibles de figurer parmi les bénéficiaires des programmes et politiques configurés de manière conventionnelle. (Voir la citation dans l'encadré à la page suivante.)

Par exemple, comme les programmes de jeunesse et autres efforts de filets de sécurité reposent sur la demande plutôt que sur le recrutement déterminé, il existe une " capture d'élite " des hommes adultes et de quelques femmes. Les filles employées comme domestiques à Addis Abeba n'ont pour ainsi dire aucun accès aux " services de jeunesse ". Et un programme de jeunesse de Monrovia (Libéria) sert près de 12 fois plus de filles de 15 à 19 ans scolarisées que de filles de 10 à 14 ans déscolarisées ${ }^{16}$ (voir le Tableau 7).

\section{TABLEAU 6 RESSOURCES DISPONIBLES}

\section{CATÉGORIES PROGRAMMATIQUES}

\section{PRÉVENTION ET RENFORCEMENT D'ACTIF-PROTECTION}

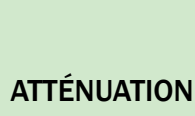

ATTÉNUATION
TRAITEMENT : ACCESSIBILITÉ ET EFFICACITÉ DE PRESTATION

RÉINTÉGRATION ET RÉTABLISSEMENT

\section{Filles vulnérables}

(filles employées comme domestiques, aînées/ enseignement primaire, cadettes/enseignement

Ressources négligeables
Ressources
négligeables
Quelques

ressources
Ressources

négligeables secondaire, filles déscolarisées de 10 à 14 ans)

\begin{tabular}{|c|c|c|c|c|}
\hline Police & $\mathrm{s} / \mathrm{o}$ & $\mathrm{s} / \mathrm{o}$ & Plus de resources & $\mathrm{s} / \mathrm{o}$ \\
\hline Avocats & $\mathrm{s} / \mathrm{o}$ & $\mathrm{s} / \mathrm{o}$ & Plus de resources & $\mathrm{s} / \mathrm{o}$ \\
\hline Juges & $\mathrm{s} / \mathrm{o}$ & $\mathrm{s} / \mathrm{o}$ & Plus de resources & $\mathrm{s} / \mathrm{o}$ \\
\hline Agents de santé & $\mathrm{s} / \mathrm{o}$ & $\mathrm{s} / \mathrm{o}$ & Plus de resources & $\mathrm{s} / \mathrm{o}$ \\
\hline Enseignants & s/o & $\mathrm{s} / \mathrm{o}$ & Plus de resources & $\mathrm{s} / \mathrm{o}$ \\
\hline $\begin{array}{l}\text { Garçons/hommes comme } \\
\text { agresseurs ou alliés poten- } \\
\text { tiels (par ex. proches intimes, } \\
\text { frères, pères, partenaires, } \\
\text { étrangers) }\end{array}$ & $\begin{array}{l}\text { Quelques } \\
\text { ressources }\end{array}$ & $\mathrm{s} / \mathrm{o}$ & $\mathrm{s} / \mathrm{o}$ & $\mathrm{s} / \mathrm{o}$ \\
\hline
\end{tabular}


TABLEAU 7 LES FILLES PLUS ÂGÉES DE L'ENSEIGNEMENT SECONDAIRE SONT PRĖS DE NEUF FOIS PLUS SUSCEPTIBLES DE PARTICIPER À UN PROGRAMME COMMUNAUTAIRE QUE LEURS CADETTES DÉSCOLARISÉES

\begin{tabular}{|c|c|c|c|c|c|}
\hline ÂGE ET TYPE & $\begin{array}{c}\text { ENS. } \\
\text { PRIMAIRE }\end{array}$ & $\begin{array}{c}\text { ENS. } \\
\text { SECONDAIRE }\end{array}$ & DÉSCOLARISÉES & MÈRES & $\begin{array}{c}\text { NON } \\
\text { MÈRES }\end{array}$ \\
\hline \multicolumn{6}{|l|}{10 à 14 ans } \\
\hline Admissibles & 1942 & 236 & 558 & $\mathrm{~s} / \mathrm{o}$ & $\mathrm{s} / \mathrm{o}$ \\
\hline$\%$ atteint & 2,3 & 5,1 & 1,4 & s/o & s/o \\
\hline \multicolumn{6}{|l|}{15 à 19 ans } \\
\hline Admissibles & 686 & 786 & 505 & 379 & 1651 \\
\hline$\%$ atteint & 7,9 & 12,0 & 7,3 & 16,6 & 7,4 \\
\hline \multicolumn{6}{|l|}{20 à 24 ans } \\
\hline Atteintes & 9 & 62 & 41 & 89 & 24 \\
\hline Admissibles & 112 & 592 & 990 & 1304 & 586 \\
\hline$\%$ atteint & 8,0 & 10,5 & 4,1 & 6,8 & 4,1 \\
\hline
\end{tabular}

SOURCE : Tabulations par Adam Weiner. EDS 2007 du Libéria et Exercice de couverture 2008-2009 du Libéria avec données pilotes de quatre programmes communautaires..

Un effort de recherche prometteur commence à définir les facteurs qui protègent les filles de la violence et les aide à voir et gérer clairement leur environnement.

Hallman constate que les réseaux d'amitié des filles sont beaucoup moins développés que ceux des garçons (généralement, en Afrique du Sud, les garçons les plus pauvres ont plus d'amis et plus de contacts réguliers avec eux que les filles les plus riches) et, observation cruciale, qu'un plus grand isolement social présente une forte corrélation avec l'expérience de rapports sexuels forcés $^{17,18}$. (Voir la Figure 2.)

Les données transversales (Lloyd) ${ }^{19}$ de nombreux pays laissent entendre que les filles scolarisées à l'adolescence (au niveau correspondant à leur âge surtout) sont moins susceptibles d'être sexuellement actives et de subir de contraintes sexuelles, d'infections de l'appareil génital, de grossesses non désirées ou de contracter le VIH. Une enquête récente menée au Swaziland démontre que le risque de la violence sexuelle durant l'enfance est le plus grand parmi les filles déscolarisées au moment de l'étude, donnant à penser que de meilleures opportunités d'instruction seraient utiles à la prévention de cette violence (Breiding et al. $)^{20}$. Les conclusions de plusieurs études laissent aussi entendre un risque supérieur parmi les filles dont la relation avec la mère biologique n'est pas étroite, et que la solidité de la relation mère-fille peut donc protéger la fille contre la violence sexuelle (Breiding et al. ${ }^{21}$. Vivre avec ses deux parents joue un rôle également protecteur. Le fait d'être orpheline, par contre, fait courir un risque significativement supérieur à la fille. Avoir une connaissance spécifique, par opposition à générale, de la communauté et de ses services représente un avantage protecteur. Les compétences de santé et celles de nature socioéconomique sont étroitement liées. Dans une étude menée en Afrique du Sud, les filles qui ont un objectif financier sont plus susceptibles d'évaluer réalistiquement leur risque de contraction du VIH et de se faire tester ${ }^{22}$.
Il existe un échec persistant à investir dans l'actif-protection des filles. Imaginez un mouvement pour les droits civils qui n'ait pas poursuivi le droit de vote des Afro-Américains. Peut-on imaginer un véritable effort en faveur de l'augmentation des salaires qui n'en appelle qu'aux patrons sans mobiliser les travailleurs?

Aucun programme ne peut rétablir les familles, mais tous peuvent assurer un soutien social, une connaissance spécifique et de nouvelles compétences. Même les filles les plus défavorisées (celles employées comme domestiques et séparées depuis longtemps de leur famille) peuvent bâtir leur capital social et leurs actif-protection de manière à améliorer leurs perspectives de vie plus sûre et acceptable. 
FIGURE 2 LES FILLES MOINS CONNECTÉES SOCIALEMENT SONT PLUS SUSCEPTIBLES DE SUBIR DES RAPPORTS SEXUELS FORCÉS

\section{(FILLES DE 14 À 16 ANS AYANT JAMAIS ÉTÉ FORCÉES}

PHYSIQUEMENT)

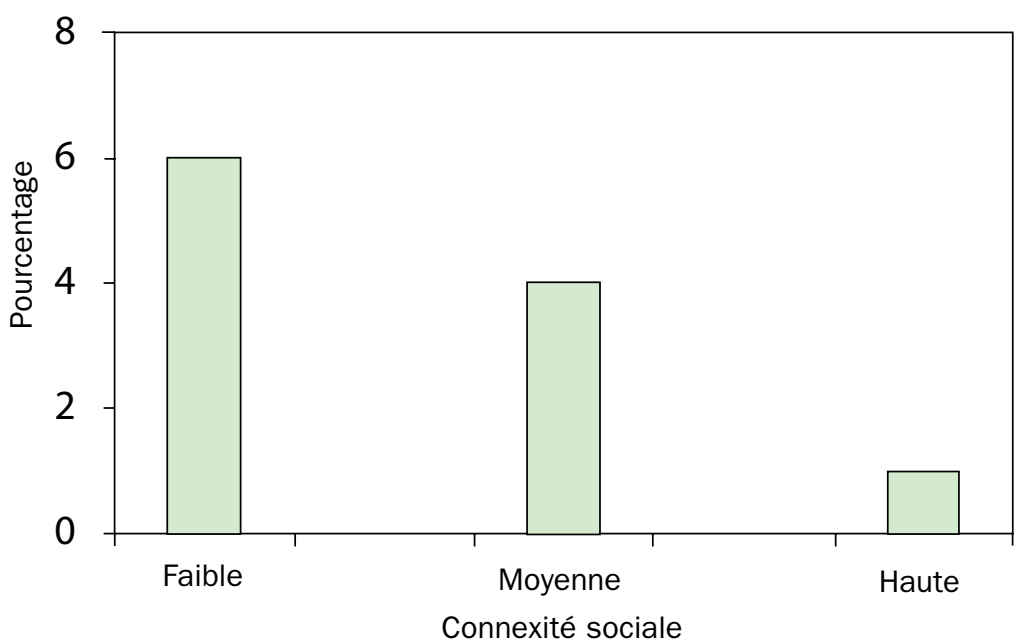

SOURCES: Hallman, K. 2004. "Socioeconomic disadvantage and unsafe sexual behaviors among young women and men in South Africa ", Policy Research Division Working Paper No. 190. New York: Population Council. Bruce, Judith et Kelly Hallman. 2008. "Reaching the girls left behind ", Gender \& Development 16(2): 227-245.

\section{POLITIQUES ET PRATIQUES PRÉCONISÉES}

Utiliser les données pour identifier les filles exceptionnellement vulnérables

Les données existent et peuvent aider à identifier les fortes concentrations spécifiques de filles et de jeunes femmes extrêmement vulnérables à la violence : par exemple, les Enquêtes à indicateurs multiples/Multiple Indicator Cluster Survey (MICS), les Enquêtes démographiques et de santé (EDS) et les enquêtes National Centers for Disease Control and Prevention (CDC) Surveys effectuées pour le Swaziland, la Tanzanie et le Zimbabwe et planifiées pour Haïti. La violence culturelle telle que le mariage précoce ou la mutilation génitale des filles se concentre souvent en des endroits géographiques ou dans des communautés ethniques/ religieuses spécifiques. On observe souvent une variation sous-nationale dans les proportions de jeunes femmes qui acceptent la violence. Par exem- ple, au Bénin ${ }^{23}$, même si la moyenne nationale des filles qui estiment que la violence conjugale est acceptable dans certaines circonstances est d'environ $45 \%$, il existe des " points chauds " où cette proportion atteint parfois jusqu'à $74 \%$ des filles. À Haïti (EDS 2006) ${ }^{24}$, la proportion des filles qui acceptent la violence dans le nord est proche du double de celles qui l'estiment justifiée dans le sud.

On observe des variations similaires dans la proportion des filles qui ont subi des rapports sexuels forcés ou d'autres circonstances qui exacerbent leur vulnérabilité, notamment la déscolarisation des filles de 10 à 14 ans, leur séparation de leurs parents ou leur emploi en tant que domestiques ${ }^{25}$. En somme, il existe suffisamment de données pour établir dans la plupart des contextes une épidémiologie fondamentale de la violence et les données existantes peuvent faciliter l'identification préalable des populations féminines particulièrement vulnérables au risque de la violence. (Voir la carte à la page 12.)
Consacrer plus de ressources au renforcement de l'actif-protection des filles les plus vulnérables

Les programmes de réduction de la violence doivent engager les intéressés à la base - en l'occurrence, les adolescentes - en leur donnant le droit à l'information et l'occasion de se réunir et d'exprimer leur point de vue. Ces droits de participation ne sont pas bien compris ou mis en œuvre et sont les plus difficiles à assurer, surtout au profit des populations marginalisées et vulnérables. Imaginez un mouvement pour les droits civils qui n'ait pas poursuivi le droit de vote des Afro-Américains. Peut-on imaginer un véritable effort en faveur de l'augmentation des salaires qui n'en appelle qu'aux patrons sans mobiliser les travailleurs?

La première nécessité est de développer l'actif-protection des filles les plus jeunes et les plus vulnérables à la violence la plus grave. Si cette valeur reçoit l'attention qu'elle mérite, les ressources pourront être affectées en majorité au renforcement de l'actifprotection des populations vulnérables tandis que d'autres appropriées mais moindres pourront l'être au niveau secondaire, notamment au renforcement des compétences professionnelles des symboles d'autorité établis ou à l'approche des populations d'agresseurs potentiels.

Même les filles les plus défavorisées peuvent améliorer leurs perspectives de vie plus sûre et acceptable.

Incorporer la connaissance des filles dans la conception des approches de prévention, d'atténuation, des procédures de déclaration et des programmes de traitement

Peu de programmes incorporent actuellement les perspectives des filles, même en leur qualité de survivantes de la violence, au niveau conceptuel et de la mise en œuvre. La grande majorité des ressources renforce le capital social et la connaissance de ceux qui 


\section{PRIORITÉS INVERSÉES}

Ceux et celles moins vulnérables, dotés d'un plus grand actif social (foyer stable, scolarisation) reçoivent la plus grande part des ressources destinées aux jeunes :

- Garçons (plus âgés) scolarisés

- Jeunes hommes non mariés

- Jeunes nés en milieu urbain, vivant avec leurs deux parents

- Adolescents plus âgés, jeunes de 20 et parfois même 24 ans et plus
Ceux et celles les plus vulnérables, dotés du moindre actif social (migrantes, familles moins stables, scolarisation moindre ou nulle, relations sexuelles non protégées les plus fréquentes) ne reçoivent qu'une part négligeable des ressources destinées aux jeunes :

- Filles (plus jeunes) déscolarisées

- Filles mariées

- Migrants, d'origine rurale, séparés de leurs parents

- Adolescents les plus jeunes, de 10 à 14 ans

SOURCE : Bruce, Judith. Novembre 2006. "Using data to count, advocate for, and invest in adolescent girls: An Ethiopian case study. " AUTRE SOURCE : Mekbib, T., A. Erulkar et F. Belete. 2005. "Who are the targets of youth programs: Results of a capacity building exercise in Ethiopia ", Ethiopian Journal of Health Development 19(1): 60-62.

exercent leur autorité sur les filles : les enseignants ou les autorités responsables de traduire en justice les auteurs de la violence (la police et les juges), notamment. Un programme national de conception récente, destiné à réduire la violence dans un pays d'Afrique subsaharienne à niveaux de violence extrêmement élevés, prévoyait des mesures d'évaluation au niveau des pairs masculins, mais pas de mesures du changement parmi les jeunes filles et femmes les plus susceptibles de subir cette violence. Les programmes de jeunesse, parmi les rares initiatives que les gouvernements conçoivent à titre préventif et pour la mise en place de "filets de sécurité ", négligent systématiquement et précisément les jeunes filles et femmes particulièrement vulnérables à la violence : celles de 10 à 14 ans déscolarisées et/ou ne vivant qu'avec un parent ou aucun et celles mineures employées dans des situations propices à l'exploitation ${ }^{26,27}$.

Le Population Council a mis au point un outil d'évaluation de la sécurité destiné aux filles et aux garçons, pour les aider à définir les moments de la journée, de la semaine ou de l'année, ou bien les situations qui représentent pour eux une menace potentielle. Les filles elles-mêmes doivent être invitées à identifier les moments où elles se sentent en danger, en fonction

- du jour de la semaine,

- de la période de l'année,

- de la situation (demande d'une note scolaire ou chemin de l'école, par exemple),

- de l'heure de la journée,

- des périodes de difficulté économique ou de plus grande facilité. Les filles peuvent être invitées à identifier les situations ou les événements particuliers qui intensifient le risque. Par exemple : les vacances scolaires, les moments où les hommes ont de l'argent, les festivals ou les événements sportifs (comme la Coupe du Monde). En Afrique du Sud, les filles ont identifié les mois de novembre et décembre comme ceux où elles ressentent le plus la pression d'échanger leurs faveurs sexuelles contre des cadeaux ou de l'argent, car il s'agit du moment où les frais de scolarité doivent être payés (Hallman) ${ }^{28}$.
Cet exercice a été mené dans plusieurs contextes, notamment au Burkina Faso, à Haïti, en Tanzanie et en Zambie. À Haïti, les filles ont établi clairement qu'il n'existe aucun moment auquel celles membres de l'une des trois catégories vulnérables (domestiques, 10 à 14 ans déscolarisées et chefs de ménage) se sentent en sécurité. L'outil d'évaluation de la sécurité jette les bases d'une discussion pratique de ce à quoi pourrait ressembler un plan de sécurité. Un extrait de l'évaluation ${ }^{29}$ met l'accent sur les périodes pouvant être sources de risques. (Voir la Figure 3.)

Créer des plates-formes et espaces régulièrement disponibles aux filles, en particulier celles les plus vulnérables à l'exploitation

Contrairement aux garçons, les filles ne disposent pas d'espaces sûrs réguliers où elles peuvent rencontrer leurs amies extérieures à la famille (une perte en soi) hors école ou foyer. Les filles défavorisées sont étrangères au foyer dans lequel elles vivent et elles ne sont pas scolarisées. D'autres filles ont quitté leur communauté, souvent de force.

Tous les êtres humains ont besoin d'amitié et d'un soutien social. Pour ce qui est des filles, ces actifs réduisent leur vulnérabilité à la violence mais peuvent aussi servir de plate-forme à l'élargissement de leurs connaissances concernant leurs droits, à l'élaboration de plans de sécurité spécifiques et à l'assurance d'espaces sûrs où elles peuvent parler des aspects sensibles et menaçants de leur vie. Une famille de pairs sous la conduite d'un mentor constitue, en soi, un atout vital. II s'agit aussi d'une filière à travers laquelle la fille peut apprendre ses droits, comprendre les codes de conduite des enseignants, avoir accès aux services et au soutien dont elle a besoin et trouver justice.

La création d'espaces régulièrement accessibles renforcera probablement les niveaux de déclaration de la violence ; les filles touchées se feront vraisemblablement une meilleure idée de ce qu'est la violence et elles l'accepteront moins. Elles se sentiront peut-être aussi plus en confiance pour 
POURCENTAGE DES FILLES DE 10 À 14 ANS NON SCOLARISÉES ET NE VIVANT AVEC AUCUN DE LEURS PARENTS AU MOZAMBIQUE (137 768)

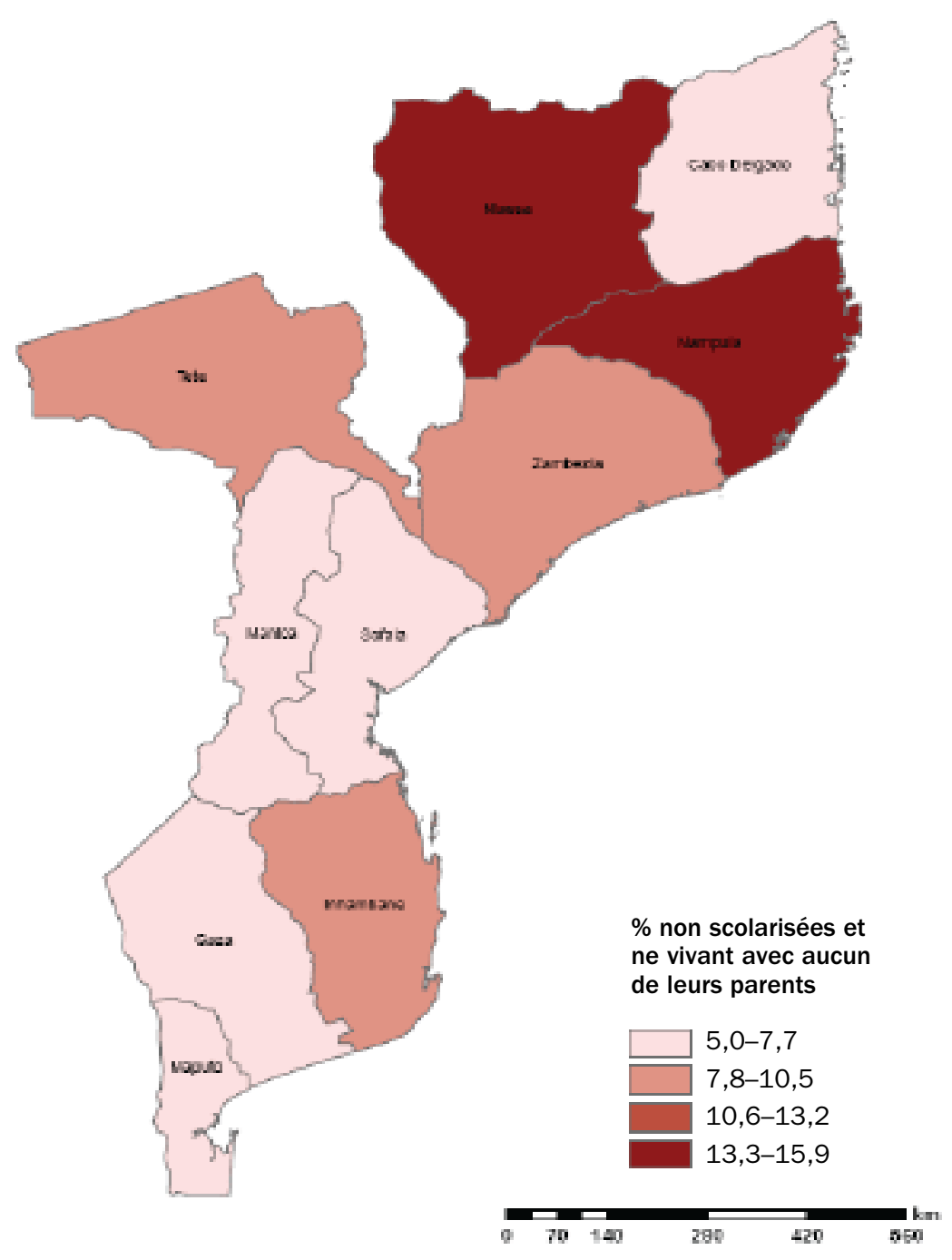

SOURCE : "The Adolescent Experience In-Depth: Using Data to Identify and Reach the Most Vulnerable Young People: Mozambique 2006. " New York : Population Council, 2009. <http://www. popcouncil.org/publications/serialsbriefs/AdolExplnDepth.asp>.

Les guides de données relatives aux adolescents du Population Council et de I'UNFPA (disponibles pour plus de 50 pays) sont accessibles sur www.popcouncil. org/girlsdata ou www.unfpa.org/ youth/dhs_adolescent_guides.html. dévoiler les situations difficiles dans lesquelles elles se trouvent ${ }^{30}$.

\section{Recruter intentionnellement les filles vulnérables}

Comme indiqué plus haut, la participation des filles les plus vulnérables (dont le risque est mesuré par l'exclusion sociale, les conditions familiales, l'emploi et la situation scolaire, la prévalence des normes culturelles et la migration, par exemple) exige leur recrutement spécifique. La cartographie de la communauté des filles est essentielle et permet l'identification de celles les plus vulnérables, notamment celles employées comme domestiques $^{31}$.

Ancrer les programmes autour des filles, en faisant d'elles les clientes principales : viser sélectivement les autres intervenants, en fonction de ce que pensent les filles

Les programmes efficaces doivent gérer de nombreux gardiens et structures restrictives pour que les filles restent leurs clientes et points d'ancrage clés. L'engagement d'autres intervenants peut être recherché (comme les autorités religieuses, les enseignants, les parents, les pairs masculins et autres figures masculines), mais les filles doivent conserver leur place centrale de clientes principales. Conformément à tout agenda de droits humains, la population affectée, en l'occurrence les filles, doivent être les participantes au cœur du programme et ses principales bénéficiaires. Dans la mesure où d'autres intervenants sont engagés au titre d'alliés, les perspectives des filles doivent éclairer la direction et les messages programmatiques.

Déterminer les médias éventuellement utiles en fonction de l'expérience des filles

On se rue souvent sur les médias dans l'idée qu'une campagne de marketing social peut réduire la violence. Or les campagnes sont souvent entreprises sans déterminer avant tout l'accès relatif des filles aux médias engagés (radio, télévision), ni d'ailleurs l'utilité du message. L'approche médiatique est souvent un moyen simple d'affecter des 


\title{
FIGURE 3 OUTIL D'ÉVALUATION DE LA SÉCURITÉ
}

\author{
PÉRIODE POUVANT ÊTRE SOURCE DE \\ RISQUES \\ RAISONS POUR LESQUELLES CETTE PÉ- \\ RIODE PEUT ÊTRE SOURCE DE RISQUES \\ CE QUI PEUT ÊTRE FAIT POUR \\ LIMITER CES RISQUES \\ Saison des récoltes \\ Saison des pluies \\ Saison de déplacement pour trouver du \\ travail \\ Frais scolaires à régler \\ Vacances \\ Période scolaire \\ (Autre) \\ (Autre) \\ (Autre)
}

Outil conceptualisé par Judith Bruce et Karen Austrian. Publié par Austrian, K. et Ghati, D. 2010. Conception d'un programme centré sur les filles : Boîte à outils pour l'élaboration, le renforcement et l'expansion de programmes destinés aux adolescentes. Population Council

ressources et de sembler défendre une cause, mais il n'est pas sûr que ces campagnes renforcent effectivement l'actif-protection des filles. De manière générale, si l'on vise le changement comportemental et, certainement, les groupes vulnérables, l'accès spécifique à l'information est beaucoup plus utile que les messages généralisés. Les filles doivent notamment reconnaître spécifiquement les scénarios risqués. Par exemple, les participantes à un atelier haïtien ont identifié les " garçons rusés " qui ciblent les filles jeunes et mal dans leur peau et les mettent dans des situations qui aboutissent souvent sur une violence collective. En Zambie, les filles ont identifié les enseignants qui insistent qu'elles les accompagnent chez eux pour recevoir une note. Ce comportement devrait être couvert dans le code des enseignants ; les filles ignorent actuellement l'existence même de ces codes car ils ne sont pas publiés ou discutés avec elles. Peut-être l'expérience la plus connue s'est-elle déroulée au Kenya, où les lycéennes ont été informées que les partenaires plus âgés sont plus susceptibles d'avoir le VIH que les plus jeunes. II s'en est suivi un changement tant au niveau des attitudes que de la sélection des partenaires $^{32}$.

\section{Protéger les filles en quête de justice}

Pour les survivantes de la violence, obtenir justice peut être une tâche difficile et qui retraumatise souvent les adolescentes. Elles se trouvent souvent confrontées à des autorités qui doutent d'elles et ignorent l'approche adéquate à adopter : on signale notamment des cas où les cliniciens soumettent les filles, même prépubères, à un examen au spéculum, qui constitue pourtant une intervention clinique dangereuse et interdite. Le mauvais traitement aux mains des prestataires médicaux implique souvent de nouvelles complications médicales et un manque d'accès aux soins nécessaires tels que la prophylaxie post-exposition éventuelle au VIH et autres infections sexuellement transmissibles ou que la contraception d'urgence pour réduire le risque de grossesse. En raison de leur âge, les filles doivent obligatoirement être accompagnées d'un gardien ou d'une personne de confiance pour de nombreux services. La justice peut s'en trouver entravée car les agresseurs sont souvent les membres de la famille ou d'autres personnes proches des familles. II peut même arriver que des adultes coupables ou complices doivent consentir au traitement médical pour que puissent commen- cer les démarches judiciaires ${ }^{33}$. Tout cela sans compter que les systèmes juridiques sont complexes et difficiles à gérer, même pour les adultes, et que de nombreux pays ne disposent pas de lois aptes à protéger et promouvoir les droits des femmes et des filles.

Le Fonds de défense judiciaire des adolescentes a défini une série de directives destinées à mieux répondre aux violations des droits des filles et à équiper l'appareil judiciaire en fonction des besoins des adolescentes. Parmi ses recommandations :

- Exiger une formation sensible au genre pour tout le personnel (police, parquet, cliniciens et juges) appelé à traiter les besoins judiciaires des adolescentes.

- Assurer des visites médicales rapides, minimalement invasives, avec peu d'exigences de déclaration.

- Adopter des mesures spéciales applicables aux adolescentes dans les tribunaux. Notamment :

- Prévoir des salles d'attente séparées pour les survivantes et les témoins, pour éviter le contact avec les agresseurs.

- Faire preuve de sensibilité lors du contre-interrogatoire pour éviter le harcèlement des survivantes. 
- Autoriser l'usage d'écrans ou les témoignages enregistrés pour éviter le contact avec les agresseurs.

- Tenir les filles informées du processus judiciaire et de ses résultats.

- Limiter le nombre de témoignages requis de la survivante.

- Imposer un délai pendant lequel la procédure judiciaire doit être entamée et conclue ${ }^{34}$.

\section{Mesurer les résultats au niveau} des filles

À en juger par les efforts programmatiques déployés jusqu'ici, il semble exister une profonde résistance à centrer, même dans un premier temps seulement, les programmes de lutte contre la violence sur le renforcement des compétences des filles elles-mêmes. Certes, l'absence de mesure du changement au niveau de la fille indique combien nous avons perdu le cap. II existe une forte tendance à mesurer les entrées (comme les messages diffusés à la radio et le nombre de policiers formés) plutôt que le changement au niveau des filles. S'il importe de documenter la minutie de la mise en $œ u v r e$, les résultats les plus clairs sont ceux visibles au niveau de la population cliente centrale.

Un indice clair de programme centré sur les filles est la présence de mesures au niveau de la fille. La recherche existante laisse entendre l'existence de plusieurs types de capital social (réseaux d'amitié, appartenance à un groupe qui se réunit régulièrement) et de filets de sécurité ${ }^{35}$ (quelqu'un vers qui se tourner en cas d'urgence ou un endroit où passer la nuit) dotés d'un rôle protecteur.

Il est d'ailleurs une raison méthodologique définitive de mesurer les résultats au niveau de la fille. À mesure de la sensibilisation des filles (et de la communauté au sens large), de nombreuses choses précédemment définies comme acceptables pourront être redéfinies comme inacceptables et déclarées violentes. Les déclarations négatives concernant la qualité des services augmentent lorsque les clientes sont informées de leurs droits ou des attentes de soins appropriées qu'elles peuvent avoir. De même, on peut s'attendre à ce que les adolescentes sensibilisées et soutenues déclarent dans un premier temps plus de violences. Les programmes ne peuvent pas garantir la sécurité d'une fille, mais ils peuvent mesurer sa préparation à faire face à la violence, moyennant l'évaluation de son actif-protection (avoir quelqu'un à qui s'adresser en cas de crise et avoir un plan de sécurité spécifique, par exemple). Les programmes peuvent fort probablement mesurer les niveaux d'aise changeants vis-à-vis de la violence au sein de la communauté et ils peuvent documenter l'expansion des espaces et des moments de la journée où les filles se sentent en sécurité. Beaucoup des mesures à court terme de l'actif-protection devraient être réalisables dans des délais relativement brefs. Par exemple, les filles peuvent obtenir une pièce d'identité et acquérir un sens plus explicite du risque au sein de leur environnement, et elles peuvent élaborer et acquérir des plans et une connaissance spécifiques pour éviter ce risque.

\section{RESSOURCES : PROGRAMIMES " LABORATOIRES D'APPRENTISSAGE ", PUBLICATIONS ET OUTILS}

Effort multiniveau de changement des normes de mariage précoce et de soutien des filles mariées : Berhane Hewan, Éthiopie ${ }^{36}$

Le programme Berhane Hewan du Population Council en Éthiopie a mesuré son succès au niveau de la fille. Le programme a évalué les niveaux de mariage précoce, l'isolement social des filles et les attitudes, ainsi que le sentiment d'aise des filles en différents endroits de la communauté et leur accès à différents degrés de participation sociale. Le programme a considéré spécifiquement les facteurs interconnectés qui placent la fille en position vulnérable au mariage d'enfant : son exclusion de l'école, son manque de réseaux d'amitié, son manque d'accès à un mentor ou à un " filet de sécurité " (quelqu'un vers qui se tourner en cas d'urgence). En même temps, le programme a engagé les gardiens et parlé activement du mariage précoce. II a incité les communautés à soutenir la scolarité régulière d'une grande proportion de filles. Pour celles qui ont dépassé l'âge de la scolarisation (souvent dès la jeune adolescence, avec peu d'instruction), des clubs de filles ont été établis et les filles déjà mariées (indépendamment de leur âge) ont participé à ces clubs une fois par semaine. Le programme a eu pour résultat de différer l'âge du mariage de deux ans, de réduire ainsi l'acceptation et la pratique d'une forme extrême de violence sexuelle et d'apporter un soutien de santé et social aux filles mariées. Pour plus d'information, voir http://www.guttmacher.org/pubs/ journals/3500609.pdf [en anglais] ou s'adresser à Annabel Erulkar, PhD (aerulkar@popcouncil.org).

Girl spaces in school: Our girls, our future (Espaces Filles dans les écoles : nos filles, notre avenir) effort de synergie pour mettre fin à la violence contre les filles en Zambie

Un programme mis en œuvre en Zambie pour renforcer spécifiquement l'actif-protection des filles. Actuellement, dans six écoles, quatre organisations différentes ont établi à titre expérimental des clubs de filles visant principalement celles les plus âgées de l'enseignement primaire et les plus jeunes de l'enseignement secondaire, car il s'agit du moment, aux alentours de la puberté, où les filles sont les plus vulnérables. Les clubs se réunissent une fois par semaine dans les locaux de l'école, sous la conduite de deux enseignantes, mères ou pairs-mentors, et couvrent un vaste programme thématique couvrant la santé, les questions sociales et l'éducation financière. Les clubs déploient aussi des boîtes à suggestions où les filles peuvent déposer leurs messages et les enseignants apprennent un code de conduite qu'ils publient ensuite 


\section{LES ESPACES SÛRS POUR LES FILLES PEUVENT OFFRIR :}

- Un espace sûr, sur lequel les filles peuvent compter en dehors du foyer ou de l'école formelle pour rencontrer leurs amies.

- Des amies : un réseau dense de pairs extérieures à la famille.

- Des mentors et des exemples à suivre, auprès desquelles les filles peuvent apprendre et qui peuvent intervenir en leur nom.

- L'expérience de faire partie d'une équipe, avec coopération et leadership.

- Alphabétisme, connaissances sur la santé, mobilité sociale : les fondements de l'autonomie.

- Apprentissage des questions d'argent et de l'épargne.

- Documentation de santé, travail et citoyenneté.

- Un endroit où réaliser et publier les évaluations de sécurité.

- Développement de plans d'autoprotection spécifique des filles et d'options de gestion de crise.

- Découverte des codes de conduite applicables aux enseignants et autres professionnels.

- Orientation et gestion des difficultés et des crises (harcèlement, menaces de mariage précoce, mutilation génitale, viol, violence).

- Accès aux droits, y compris en ce qui concerne le VIH.

- Planification des périodes de stress, comme le moment de payer les frais de scolarité et les périodes de pénurie alimentaire, qui accroissent souvent la pression d'accorder ses faveurs sexuelles en échange de cadeaux ou d'argent.

- Gestion des situations de maladie prolongée, décès, héritage, planification de succession.

- Participation, activité, loisirs.

Bruce, Judith. " Reaching The Girls Left Behind: Targeting Adolescent Programming for Equity, Social Inclusion, Health, and Poverty Alleviation. " Avril 2007. Préparé pour " Financing Gender Equality: A Commonwealth Perspective ", Commonwealth Women's Affairs Ministers' Meeting, Ouganda, juin 2007.

pour que les filles (et autres intéressés) puissent en être informés. Une fille doit savoir, tout comme un enseignant, qu'il est inacceptable pour celui-ci de demander à une élève de se rendre chez lui pour obtenir une note ou d'engager avec elle une relation personnelle en dehors de l'école. Ce programme est innovant en ce qu'il a franchi le pas, passant de la concentration sur le viol et la réparation à la prévention et à l'engagement de jeunes hommes identifiés dans des contextes parallèles pour travailler avec les filles et maximiser les bienfaits synergiques de l'initiative.

Pour plus d'information, s'adresser à Caroline Muthoni Muriithi (cmuriithi@ equalitynow.org).
Promotion d'espaces de travail sûrs pour les femmes employées dans les bars: Tanzanie

Le projet TAMASHA, en cours à Dar es Salaam et en déploiement à Arusha, cherche à résoudre la vulnérabilité des jeunes femmes (officiellement âgées de 18 à 24 ans mais parfois aussi jeunes que 16 ans et moins) employées dans les bars. Ces jeunes femmes gagnent bien moins que le salaire minimum, dans un contexte où les propriétaires, leurs collègues masculins et les clients attendent d'elles qu'elles se soumettent au harcèlement sexuel (caresses, claques, etc.), sous peine de perdre leur emploi. La plupart des propriétaires n'ont pas l'intention d'assurer la couverture de ces jeunes femmes sous des politiques du travail telles qu'assurance médicale, protection contre les abus et le harcèlement, législation relative au salaire minimum ou même prévention du VIH sur les lieux du travail. TAMASHA crée un réseau visant à accroître le soutien social de ces jeunes femmes et pourrait, en vertu de la législation tanzanienne, inclure un syndicat des serveuses et employées de bar pour protéger leurs droits et négocier des conditions de travail et une protection appropriées. Les réunions bimensuelles des filles et jeunes femmes leur donnent l'occasion d'explorer ensemble leurs conditions de vie dans un milieu accueillant. D'autres intéressés, comme les propriétaires de bar, seront également invités aux conversations en vue de définir des normes de traitement et salaire minimum dans ce secteur risqué. Les membres lutteront individuellement et collectivement en faveur d'un environnement sûr, sans harcèlement, et de conditions de travail acceptables.

Pour plus d'information, s'adresser à Richard Mabala (rmabala@yahoo.com).

Biruh Tesfa (Avenir prometteur) : Offrir des espaces de rencontre, renforcer les compétences et l'actifprotection des filles employées comme domestiques, orphelines et migrantes en Éthiopie urbaine

Ce projet - produit d'une collaboration entre le Population Council, la Commission pour les jeunes et les sports d'Addis Abeba et le ministère éthiopien 


\section{MESURES DESCRIPTIVES ÉLÉMENTAIRES DU CADRAGE DE CAPITAL SOCIAL ET RISQUE DE VIOLENCE POTENTIEL DES FILLES}

- Âge/documentation d'âge

- Conditions de logement

- Situation scolaire

- État matrimonial

- Maternité

- Situation migratoire

- Travail rémunéré et non rémunéré

- Expérience de l'épargne

- Densité des réseaux d'amitié

- Accès régulier à un lieu de rencontre de pairs extérieures à la famille

- Niveaux d'affiliation sociale/appartenance à un groupe

- Niveau d'aise déclaré à l'école et dans la communauté

- Situation d'activité sexuelle (si possible) - première expérience sexuelle forcée ou amenée par la ruse

de la Jeunesse et des Sports - a déjà atteint quelque 20000 filles dans six villes d'Éthiopie, parmi la population féminine déscolarisée la plus vulnérable. Les filles se rencontrent dans les salles et espaces communautaires existants que leur offrent les administrations locales. Trois à cinq réunions sont organisées par semaine. Biruh Tesfa vise à résoudre le problème de l'isolement social des filles en renforçant leur participation et leur capital social et en leur donnant accès à une instruction et à des compétences de vie élémentaires, à l'information sur le VIH et la santé génésique et à la connaissance spécifique des ressources disponibles dans leur zone d'influence. Le projet remet à toutes ses participantes une carte d'identité revêtue de leur photo. Une évaluation réalisée en 2009 a déterminé qu'il est possible de créer des réseaux sociaux et des filets de sécurité pour les filles. Les filles inscrites au programme font état de réseaux d'amitié plus nombreux dans leurs quartiers et disent avoir des espaces où rencontrer d'autres filles en dehors de chez elles. Les participantes sont significativement plus susceptibles d'avoir accepté un conseil et dépistage volontaire du VIH que les filles du groupe témoin ${ }^{38}$.

Pour plus d'information, s'adresser à Annabel Erulkar (aerulkar@popcouncil.org).

\section{Abriendo Oportunidades : Espaces} sûrs au sein d'un programme destiné aux filles du Guatemala rural

Le programme Abriendo Oportunidades du Population Council, en collaboration avec les clubs de filles indigènes des hauts-plateaux mayas du Guatemala rural, a mis au point une approche d'" évasion en espace sûr " aujourd'hui testée à titre pilote dans trois des 40 villages du programme. L'approche consiste à faciliter les stratégies centrées sur les adolescentes, afin de prévenir et de résoudre le problème de la violence à l'encontre des filles. Avec l'aide de mentors, de jeunes leaders féminines (âgées de 15 à 24 ans) appliquent la technologie GPS pour cartographier leur communauté. Elles commencent par définir les limites de la communauté (certains villages sont ainsi cartographiés pour la toute première fois), puis vont de porte à porte pour identifier les filles admises à participer au programme.

Durant l'exercice de cartographie, les filles (de 12 à 15 ans) désignent les endroits de la communauté, les points de service et les établissements qu'elles définissent comme "sûrs ", " plus ou moins sûrs " et "non sûrs ". Elles présentent ensuite leurs cartes à une représentation transversale de la communauté (quelques anciens, les membres du comité de développement, les enseignants, des hommes plus âgés et plus jeunes et les membres intéressés des familles). Les participants voient ainsi le monde à travers les yeux des filles, notamment en ce qui concerne ses paramètres de sécurité. Les itinéraires journaliers des filles sont souvent superposés sur les cartes des villages (le chemin de l'école, par exemple). Les points incertains pour la sécurité des filles (champs en friche, lieux de rencontre des hommes, relais routiers) sont marqués. Dans certains cas, les filles ont marqué sur leur carte les endroits précis où des actes de violence ont été commis - guidant par là implicitement leurs décisions de mobilité. Cet exercice est le catalyseur d'un processus communautaire explicite. Notamment, il en résulte généralement une décision des filles de se rendre à l'école en groupes. Dans une communauté, une commission sur la sécurité a été établie.

Pour plus d'information, s'adresser au consultant du Population Council Angel del Valle (adelvalle@popcouncil.org).

\section{Contrat de communauté contre la} violence : Tostan, Afrique occidentale Le programme Tostan s'est fait le pionnier d'une forme de dialogue avec les communautés dans le but de rejeter la mutilation génitale des filles et, plus récemment, le mariage précoce. Bien que qu'il ne mesure pas encore ses résultats au niveau de la fille, ce programme s'est fait le catalyseur d'efforts communautaires destinés à éliminer les formes de maltraitance 
grave. Une stratégie de principe utilisée à ces fins est conçue pour accélérer l'abandon de la mutilation génitale des filles. Dans les villages qui ont participé au programme Tostan, la prévalence de la mutilation génitale des filles aurait baissé de $70 \%$ par rapport à $40 \%$ dans les villages témoins (d'après les déclarations de "témoins privilégiés ", pas des filles). Cette stratégie repose sur la notion que pour changer les normes sociales, il faut atteindre une masse critique de membres de la communauté, car la mutilation génitale et le mariage précoce des filles sont liés à une norme généralement perçue de la fille considérée "décente " pour le mariage. L'approche Tostan recourt à l'évaluation qualitative pour améliorer la force de ses prestations ${ }^{39}$.

Pour plus d'information, s'adresser à Gannon Gillespie, directeur au développement stratégique, Tostan (gannongillespie@tostan.org ; tél. +1-212-2991156).

Programme Safe Cities : The Association for the Development and Enhancement of Women (association pour le développement et l'amélioration de la condition féminine), Égypte

Le programme Safe Cities, aujourd'hui déployé dans plusieurs quartiers du Caire, cherche à réduire le harcèlement et la violence sexuelle à l'encontre des filles et des femmes dans les espaces urbains publics (places de quartier, allées, immeubles abandonnés, chantiers). Le harcèlement sexuel est considéré comme empêchant les filles et les femmes de mener à bien leurs responsabilités domestiques, d'accéder aux services et à l'emploi, de développer leurs compétences, comme entravant leur accès à l'école et comme suscitant un manque de sécurité dans les jardins publics. Le projet s'adresse aux femmes et aux filles vulnérables, aux autorités locales, à d'autres groupes locaux et aux médias, dans le but d'améliorer l'accès et la sécurité dans les espaces publics.

Pour plus d'information, s'adresser à Iman Bibars (ibibars@ASHOKA.ORG),

\section{MESURES PLUS SPÉCIFIQUES POUR ÉVITER LES MENACES POTENTIELLES EXISTANTES}

- Filets de sécurité

- Quelqu'un à qui s'adresser en cas d'urgence

- Endroit où passer la nuit en cas d'urgence

- Quelqu'un à qui emprunter de l'argent en cas d'urgence

- Estime personnelle/sens de soi/aspirations

- Documentation personnelle/reconnaissance d'appartenance à la communauté

- Connaissance contextuelle des services (lieu, heures, preuve d'inscription)

- Connaissance spécifique des personnes, périodes et événements risqués

- Plans de sécurité/urgence spécifiques

SOURCES : Erulkar, Annabel S. et Muthengi, Eunice. 2008. "Evaluation of Berhane Hewan: A program to delay child marriage in rural Ethiopia. International Perspectives on Sexual and Reproductive Health " 35(1): 6-14. Erulkar, Annabel S., Mekbib, Tekle-Ab, Tegegne, Mesfin. 2008. "Biruh Tesfa: Creating a " Bright Future " for migrant girls in urban areas of Ethiopia. " Promoting Healthy, Safe, and Productive Transitions to Adulthood Brief. Bruce, Judith. 2003. Adolescent and Youth Sexual and Reproductive Health: Charting Directions for a Second Generation of Programming. Background Documents for the Meeting, pp. 77-89. Bruce, Judith ; Hallman, Kelly. 2008. "Reaching the girls left behind." Gender and Development 16(2): 227-245.

à l'Association for the Development and Enhancement of Women.

\section{Paraprofessional Social Work}

Training Program (programme de formation paraprofessionnelle au travail social), International Rescue Committee (IRC), Région Somali d'Éthiopie

Le programme de formation Paraprofessional Social Work vise les filles et les femmes vivant dans les districts difficiles d'accès de la région Somali d'Éthiopie. Avec l'aide de l'UNICEF, ce programme engage deux membres de la communauté de chaque village (une femme et un homme) dans une formation d'une année pour en faire des assistants sociaux. Ces personnes sont issues de villages où le conflit est endémique et où les filles subissent probablement de hauts niveaux de violence sexuelle, psychologique et physique sous l'effet du conflit et des normes culturelles traditionnelles de la région Somali. Au fil de l'année, dans le cadre d'un programme de travail social plus vaste, I'IRC offre à ses étudiants une série de quatre formations d'une semaine sur la réponse à la violence de genre. Les sujets abordés incluent la gestion de cas, l'apport d'une assistance psychosociale générale, l'écoute active, la confidentialité et l'orientation. Les candidats assistants sociaux se rencontrent chaque trimestre dans la capitale régionale aux fins de leur formation et passent le reste du temps à pratiquer leurs nouvelles compétences sur le terrain. Le programme de formation psychosociale s'accompagne d'un programme de formation aux questions de justice, santé et service juridique destiné à renforcer les capacités des prestataires de ces autres secteurs à répondre aux besoins des survivantes 
de la violence. La phase suivante du projet prévoit le développement d'une procédure opérationnelle standard destinée aux prestataires, dans le but d'établir les rôles et responsabilités de chaque secteur, d'élaborer une filière d'orientation et de rationaliser le processus de réponse. Ce programme aide les filles et les femmes à accéder à l'assistance dont elles ont besoin, qu'elles aient subi des actes ou menaces de violence ou qu'elles recherchent simplement une connexion sociale. Dans un contexte libre de conflit offrant un meilleur accès aux populations, cette intervention pourrait être plus complète. Ce modèle est cependant utile dans les zones de conflit où les organismes humanitaires éprouvent des difficultés à accéder à la population ciblée.

Pour plus d'information, s'adresser à Jody Myrum (Jody.Myrum@nike.com).

Growing Up Safe and Healthy (SAFE) : Combler l'inégalité de genre par les messages de prévention de la violence de genre au Bangladesh

Le projet Growing Up Safe and Healthy (SAFE) vise à promouvoir l'égalité de genre pour les filles mariées et non mariées de 10 à 17 ans vivant dans les bidonvilles urbains du Bangladesh. SAFE cherche à résoudre la vulnérabilité et la marginalisation intenses des adolescentes en ciblant les problèmes du mariage précoce et de la violence de genre à travers des programmes de sensibilisation aux violations des droits des femmes, par le biais d'une combinaison de messages de santé sexuelle et génésique (intégrité du corps, questions de consentement et de choix et promotion générale de l'autodétermination). SAFE établit aussi de solides liens et réseaux entre les services judiciaires et les prestataires de services de santé sexuelle et génésique situés à proximité des bidonvilles, les défenseurs des droits humains et des droits des femmes, les organisations de recherche et le ministère bangladais de la Condition féminine et des enfants. Aux centres de services juridiques et de santé, le per- sonnel de SAFE instruit les filles et les femmes sur la manière de se protéger des risques de santé sexuelle et génésique et de la violence de genre.

Pour plus d'information, s'adresser à Sajeda Amin (samin@popcouncil.org).

\section{Apne Aap: Éliminer le trafic sexuel en Inde}

Fondée par la journaliste d'investigation Ruchira Gupta, Apne Aap est une organisation de base établie à Mumbai, en Inde (avec d'autres sites implantés à Bhiwandi, au Bihar, à Delhi et au Bengal-Occidental), qui cherche à atteindre les femmes et les enfants les plus isolés et les plus vulnérables sous l'emprise ou la menace de la prostitution dans les quartiers rouges et les bidonvilles de l'Inde. Apne Aap mobilise et guide des groupes communautaires de femmes et filles trafiquées et vulnérables pour qu'elles s'autonomisent les unes les autres. Apne Aap offre un espace sûr et des occasions de formation juridique, éducative et de subsistance aux filles et aux femmes, pour qu'elles puissent développer les compétences et capacités de résister aux trafiquants. L'organisation a atteint plus de 10000 femmes et filles ; 812 d'entre elles sont scolarisées dans des écoles ordinaires, 1200 ont fondé de petites entreprises coopératives appelées groupes d'entraide et 3042 ont soumis au parlement indien une requête d'amendement de la loi pour la prévention du trafic immoral (ITPA) en vue de punir les acheteurs et de protéger les femmes et les filles.

Pour plus d'information, s'adresser à Zoe Young (zoe@apneaap.org).

\section{Un SEUL programme : Approche} pédagogique unifiée de la sexualité, du genre, du VIH et des droits humains

\section{Un SEUL programme offre une res-} source à l'élaboration d'un programme scolaire unifié sur la sexualité, le genre, le VIH et les droits humains. Inspiré de la recherche mondiale sur les risques posés à la santé sexuelle, l'ouvrage répond, entre autres mandats, à l'appel des Objectifs du Millénaire pour le développement et il propose une approche pratique inédite et fraîche à l'éducation des jeunes dans un monde divers et changeant. Plus spécifiquement, Un SEUL programme aide les éducateurs et les décideurs à envisager non seulement les déterminants individuels de la santé sexuelle et génésique des jeunes, mais aussi les besoins distinctifs de différents sousgroupes - filles, garçons, homosexuels et lesbiennes. L'élaboration d'Un SEUL programme a été confiée à un groupe d'experts internationaux dans le but d'aider les jeunes à s'assumer et à affirmer et défendre leur droit à la dignité, à l'égalité et à une vie sexuelle saine, satisfaisante et responsable.

Un SEUL programme peut être téléchargé (format PDF) en français, en anglais ou en espagnol, ou un exemplaire imprimé peut être commandé sur <www.ItsAllOne.org>.

\section{Autres ressources:}

Amin, Sajeda et Althea D. Anderson. Mai 2011. "Addressing sexual and genderbased violence (SGBV) against adolescent girls," Promoting Healthy, Safe, and Productive Transitions to Adulthood Brief, no. 38. <http://www.popcouncil. org/pdfs/TABriefs/38_SGBV.pdf > [en anglais].

Krause-Vilmar, Jina. 2011. Preventing Gender-based Violence, Building Livelihoods: Guidance and Tools for Improved Programming. New York: Women's Refugee Commission. < womensrefugeecommission.org/resources/ doc_download/798-preventing-genderbased-violencebuilding-livelihoods-guidance-and-toolsfor-improved-programming> [en anglais].

Ricardo, C., G. Barker et M. Eads. 2012, à paraître. Engaging Boys and Young Men in the Prevention of Sexual violence: $A$ Systematic and Global Review of Evaluated Interventions. SVRI et Promundo [en anglais].

Together for Girls est un partenariat mondial du secteur public et privé voué à l'élimination de la violence sexuelle à l'encontre des filles. Ce partenariat 
se concentre sur trois grands axes : mener et soutenir des enquêtes nationales sur l'ampleur et l'impact de la violence à l'encontre des enfants, axées en particulier sur la violence sexuelle à l'encontre des filles ; appuyer les initiatives programmatiques coordonnées en réponse aux données : et mener des efforts mondiaux de plaidoyer et de sensibilisation de l'opinion pour attirer l'attention sur le problème et promouvoir des solutions factuelles. Contact : Jennifer Kim (jen@togetherforgirls.org). Site Web : <www. togetherforgirls.org>.

\section{NOTES}

1 Ellsberg, M. et L. Heise. Researching Violence against Women: A Practical Guide for Researchers and Activists. Washington, DC: World Health Organization, 2005. [En anglais.]

2 "Violence against children in Tanzania: Findings from a national survey 2009. " Fonds des Nations Unies pour l'enfance, US Centers for Disease Control and Prevention, Muhimbili University of Health and Allied Sciences, août 2011. [En anglais.]

3 Reza, Avid et al. "Sexual violence and its health consequences for female children in Swaziland: A cluster survey study." US Centers for Disease Control and Prevention, 2009. [En anglais.]

4 Voir la note 2.

5 Gray, Ron, Fred Nalugoda, David Serwadda et Maria Wawer. "Marriage and HIV risk: Data from Rakai, Uganda. " Document présenté à la rencontre Exploring the Risks of HIV/AIDS Within the Context of Marriage, Population Council, New York, 10 novembre 2004. [En anglais.]

6 Jejeebhoy, Shireen. "The adverse health and social outcomes of sexual coercion: Experiences of young women in developing countries. " Population Council, 2004. [En anglais.]

7 Garcia-Moreno, Claudia. " Putting women first: Ethical and safety recommendations for research on domestic violence against women. " Department of Gender and Women's Health, World Health Organization. Geneva, Switzerland, 2001. [En anglais.]
8 "Violence against children in Tanzania: Findings from a national survey 2009." Fonds des Nations Unies pour l'enfance, US Centers for Disease Control and Prevention, Muhimbili University of Health and Allied Sciences, août 2011. [En anglais.]

9 Pour une discussion plus complète, voir aussi Hallman 2004 (Afrique du Sud); Erulkar et al. 2004a ; 2004b (Éthiopie); Amin et al. 2002 (Bangladesh); Haberland et al. 2003 (Inde) ; UNICEF et Population Council 2002 ; et Alexander et al. 2006. Les enquêtes National Surveys of Adolescence réalisées par le Guttmacher Institute et ses partenaires au Burkina Faso, au Ghana, au Malawi et en Ouganda ont produit des données sur la densité des réseaux d'amitié, indiquant dans tous les groupes (ruraux/ urbains, niveau de scolarité atteint) que les garçons ont en moyenne plus d'amis proches que les filles. (A. Biddlecom correspondance 2008 avec le responsable de la recherche.)

10 Maternowska, Catherine et Ouedraogo, Ghislaine. "Haiti Adolescent Girl Network \& Espas Pa Mwen: A Report on Adolescent Girl Programming in Haiti." Juillet 2010.

11 Pour une discussion plus complète, voir aussi Hallman 2004 (Afrique du Sud) ; Erulkar et al. 2004a, 2004b (Éthiopie) ; Amin et al. 2002 (Bangladesh) ; Haberland et al. 2003 (Inde); UNICEF et Population Council 2002 ; et Alexander et al. 2006. Les enquêtes National Surveys of Adolescence réalisées par le Guttmacher Institute et ses partenaires au Burkina Faso, au Ghana, au Malawi et en Ouganda ont produit des données sur la densité des réseaux d'amitié, indiquant dans tous les groupes (ruraux/ urbains, niveau de scolarité atteint) que les garçons ont en moyenne plus d'amis proches que les filles. (A. Biddlecomcorrespondance 2008 avec le responsable de la recherche.)

12 Une équipe du Population Council (Bruce, Austrian) a mis au point un outil d'évaluation de la sécurité utile dans le contexte des ateliers pour commencer à définir les paramètres de la sécurité durant la journée, la semaine et selon le contexte. Une introduction à ces paramètres est proposée dans la boîte à outils accessible sur http://www. popcouncil.org/publications/ books/2011_AdolGirlsToolkit_fr.asp.
13 Simbaya, Joseph et Martha Brady. 2009. "Understanding adolescent girls' protection strategies against HIV: An exploratory study in urban Lusaka ". Population Council. [En anglais.]

14 Campbell et al. 2002 ; Gazaramian et al. 1995 ; Goldin 1996; Murphy et al. 2001; Zierler et al. 1991.

15 Reza, Avid et al. 2009. "Sexual violence and its health consequences for female children in Swaziland: A cluster survey study. " Center for Disease Control, [En anglais.]

16 Source : EDS 2007 du Libéria et Exercice de couverture 2008-09 du Libéria avec données pilotes de quatre programmes communautaires. Tabulations par Adam Weiner.

17 Hallman, Kelly. 2010. " Adolescent social exclusion: The gendering of HIV risk in KwaZulu-Natal, South Africa. " Population Council, [En anglais.]

18 Bruce, Judith et Kelly Hallman. 2008. "Reaching the girls left behind ", Gender \& Development 16(2): 227-245. [En anglais.]

19 Lloyd, Cynthia. " Growing Up Global: The Changing Transitions to Adulthood in Developing Countries. " National Academies Press, 2005. [En anglais.]

20 Breiding, Matthew J. et Avid Reza. 2011. "Risk factors associated with sexual violence towards girls in Swaziland. " Bulletin of the World Health Organization 89: 203-210. [En anglais.]

21 Breiding, Matthew J. et Avid Reza. 2011. "Risk factors associated with sexual violence towards girls in Swaziland. "Bulletin of the World Health Organization 89: 203-210. (2) Lesniak, L.P. 1993. "Penetrating the conspiracy of silence: Identifying the family at risk for incest. " Family Community Health 16: 66-76. (3) Ajuwon, A.J., A. Olaleye, B. Faromoju et O. Ladipo. 2006. "Sexual behavior and experience of sexual coercion among secondary school students in three states in North Eastern Nigeria. "BMC Public Health 6: 310. doi:10.1186/1471-2458-6-310 PMID:17187685. (4) Boney-McCoy, S. et D. Finkelhor. 1995. "Prior victimization: A risk factor for child sexual abuse and for PTSD-related symptomatology among sexually abused youth. " Child Abuse \& Neglect 19: 1401-1421. [En anglais.] 
22 Hallman, K. et al. 6 juin 2007. "Social capital, socioeconomic aspirations, and HIV risk behaviors among poor South African youth. "Affiche présentée à la $3^{\mathrm{e}}$ Conférence sud-africaine sur le sida à Durban (Afrique du Sud).

23 "Étude approfondie sur les adolescents : Exploiter les données pour identifier et atteindre les jeunes les plus vulnérables : Bénin 2006. " New York: Population Council, 2009 (Page 49).

24 "The Adolescent Experience In-Depth: Using Data to Identify and Reach the Most Vulnerable Young People: Haiti 2005/06. "New York: Population Council, 2009 (Page 49). [En anglais.]

25 Erulkar, Annabel. " Adolescent Life in Low Income and Slum Areas of Addis Ababa Ethiopia. "Population Council, 2004. [En anglais.]

26 Bruce, Judith et Kelly Hallman. 2008. "Reaching the girls left behind ", Gender \& Development 16(2): 227-245. [En anglais.]

27 Liberia Institute of Statistics and GeoInformation Services (LISGIS). 2009. 2008 Population and Housing Census Final Results. Monrovia: LISGIS. Lloyd, Cynthia B. (ed.). 2005. Growing Up Global: The Changing Transitions to Adulthood in Developing Countries. Washington, DC: National Academies Press. Mekbib, T., A. Erulkar et F. Belete. 2005. "Who are the targets of youth programs: Results of a capacity building exercise in Ethiopia ", Ethiopian Journal of Health Development 19(1): 60-62. Macro International. 2011. MEASURE DHS STATcompiler. <http://www. measuredhs. com>. Site consulté le 8 mars 2011. Population Council. 2006. Comment mener un exercice de couverture : Outil d'évaluation rapide de programmes et services. New York : Population Council. Population Council. 2009. The Adolescent Experience In-Depth: Using Data to Identify and Reach the Most Vulnerable Young People (Malawi 2004). New York : Population Council. Division de la population du Département des affaires économiques et sociales du Secrétariat de l'ONU, World Population Prospects: The 2008 Revision, <http:// esa.un.org/ unpp>. Site consulté le 2 février 2011. Weiner, Adam. 2010. "Geographic variations in inequities in access to sexual and reproductive health services ", Studies in Family Planning 41(2): 134-138. [En anglais sauf références indiquées en français.]

28 Hallman 2004, communication personnelle.

29 Conceptualisée par Judith Bruce et Karen Austrian. Publiée par Austrian, K. et D. Ghati. Conception d'un programme centré sur les filles : Boîte à outils pour l'élaboration, le renforcement et l'expansion de programmes destinés aux adolescentes. New York : Population Council, 2010.

30 Pour un examen de l'importance du capital social, voir : Bruce, Judith et Kelly Hallman. 2008. "Reaching the girls left behind ", Gender \& Development 16(2): 227-245. Pour une description des étapes nécessaires à la création de plates-formes pour les filles, voir Austrian, K. et D. Ghati. Conception d'un programme centré sur les filles: Boîte à outils pour l'élaboration, le renforcement et l'expansion de programmes destinés aux adolescentes. New York : Population Council, 2010. [En anglais sauf références indiquées en français.]

31 Catino, Jennifer, Alejandra Colom et Marta Julia Ruiz. 2011. "Equipping Mayan girls to improve their lives ", Transitions to Adulthood Brief no. 5, http://www. popcouncil.org/pdfs/ TABriefs/05_MayanGirls.pdf ; Erulkar, Annabel, Belaynesh Semunegus et Gebeyehu Mekonnen. 2011. "Biruh Tesfa provides domestic workers, orphans, and migrants in urban Ethiopia with social support, HIV education, and skills ", Transitions to Adulthood Brief no. 21, <http:// www.popcouncil.org/pdfs/ TABriefs/21_ BiruhTesfa.pdf $>$. [En anglais.]

32 Dupas, Pascaline. 2010. Do Teenagers Respond to HIV Risk Information? Evidence from a Field Experiment in Kenya. Manuscrit non plublié, UCLA. [En anglais.]

33 Jody Myrum, Nike Foundation, communication personnelle.

34 Fonds de défense judiciaire des adolescentes. À paraître. " Equality Now Adolescent Girls Legal Defense Fund Learning from Cases of Girls' Rights. " Pour plus de détails, s'adresser à Mehr Qureshi (mqureshi@equalitynow.org).
35 Erulkar, Annabel. " Adolescent life in low income and slum areas of Addis Ababa Ethiopia. " Population Council, 2004. [En anglais.] Hallman, K. et al. "Social capital, socioeconomic aspirations, and HIV risk behaviors among poor South African youth. "Affiche présentée à la $3^{e}$ Conférence sud-africaine sur le sida à Durban (Afrique du Sud), le 6 juin 2007.

36 Erulkar, Annabel S. et Eunice Muthengi. 2009. "Evaluation of Berhane Hewan: A program to delay child marriage in rural Ethiopia. " International Perspectives on Sexual and Reproductive Health 35(1): 6-14. [En anglais.]

37 Programme CRISP au Kwazulu-Natal.

38 Erulkar, Annabel. "Biruh Tesfa (Bright Future) Program Provides Domestic Workers, Orphans \& Migrants in Urban Ethiopia with Social Support, HIV Education \& Skills. " Population Council. [En anglais.]

39 Diop, Nafissatou, Amadou Morwau et Helene Benga. 2008. "Evaluation of the long-term impact of the TOSTAN programme on the abandonment of FGM and early marriage: Results from a qualitative study in Senegal ", Rapport final de FRONTIERS. New York: Population Council. [En anglais.]

\section{REMERCIEMENTS}

Ce compte rendu a été rédigé [en anglais] par Judith Bruce, Senior Associate et Policy Analyst au Population Council Le projet a bénéficié de l'aide immense de nombreux intervenants. L'auteur tient à exprimer sa gratitude à ses collègues et collaborateurs pour leurs précieuses critiques et contributions techniques: Sajeda Amin, Farida Deif, Dina Deligiorgis, Lori Heise, Heidi Lehmann, Michele Moloney-Kitts, Jody Myrum et Avid Reza. Merci à Nicole Ippoliti, Sura Rosenthal, Gina Duclayan et Christina Tse pour leur assistance intarissable à la rédaction et à la production. L'auteur est seule responsable des erreurs ou omissions éventuelles. L'auteur tient enfin à remercier le Population Council, la Nike Foundation, la Packard Foundation et l'Équipe spéciale des Nations Unies pour les adolescentes pour leur partenariat et leur appui : sans eux, la réalisation de ces comptes rendus ne serait tout simplement pas possible. 



\section{Population Council}

Le Population Council s'attelle aux questions cruciales de la santé et du développement - qu'il s'agisse d'endiguer la progression du VIH, d'améliorer la santé reproductive ou d'offrir aux jeunes la promesse d'une vie satisfaisante et productive. Par ses travaux de recherche en biomédecine, en sciences sociales et en santé publique dans 50 pays, il s'efforce d'apporter avec ses partenaires des solutions qui conduisent à des politiques, des programmes et des technologies plus efficaces, pour améliorer la vie partout dans le monde. Fondé en 1952, le Population Council est une organisation non gouvernementale à but non lucratif. II siège à New York sous la conduite d'un conseil d'administration international.

www.popcouncil.org 